\title{
Developmentally Transient CB1Rs on Cerebellar Afferents Suppress Afferent Input, Downstream Synaptic Excitation, and Signaling to Migrating Neurons
}

\author{
Jesse L. Barnes, Claudia Mohr, Caitlin R. Ritchey, Chloe M. Erikson, ${ }^{\star}$ Hiroko Shiina, ${ }^{\star}$ and David J. Rossi \\ Washington State University, Integrative Physiology and Neuroscience, Pullman, Washington 99164
}

\begin{abstract}
The endocannabinoid system plays important roles in brain development, but mechanistic studies have focused on neuronal differentiation, migration, and synaptogenesis, with less attention to transcellular interactions that coordinate neurodevelopmental processes across developing neural networks. We determined that, in the developing rodent cerebellar cortex (of both sexes), there is a transient window when the dominant brain cannabinoid receptor, CB1R, is expressed on afferent terminals instead of output neuron Purkinje cell synapses that dominate the adult cerebellum. Activation of these afferent CB1Rs suppresses synaptic transmission onto developing granule cells, and consequently also suppresses excitation of downstream neurons in the developing cortical network, including nonsynaptic, migrating neurons. Application of a CB1R antagonist during afferent stimulation trains and depolarizing voltage steps caused a significant, sustained potentiation of synaptic amplitude. Our data demonstrate that transiently expressed afferent CB1Rs regulate afferent synaptic strength during synaptogenesis, which enables coordinated dampening of transcortical developmental signals.
\end{abstract}

Key words: cannabinoid; cerebellum; development; marijuana; migration; THC

\section{Significance Statement}

The endogenous cannabinoid system plays diverse roles in brain development, which, combined with the rapidly changing legal and medical status of cannabis-related compounds, makes understanding how exogenous cannabinoids affect brain development an important biomedical objective. The cerebellum is a key brain region in a variety of neurodevelopmental disorders, and the adult cerebellum has one of the highest expression levels of CB1R, but little is known about CB1R in the developing cerebellum. Here we report a developmentally distinct expression and function of CB1R in the cerebellum, in which endogenous or exogenous activation of CB1Rs modifies afferent synaptic strength and coordinated downstream network signaling. These findings have implications for recreational and medical use of exogenous cannabinoids by pregnant and breastfeeding women.

\section{Introduction}

Laws and societal attitudes about cannabinoids (including cannabis and plant-derived and related synthetic psychoactive compounds) are evolving more rapidly than our understanding of

\footnotetext{
Received Aug. 7, 2019; revised June 15, 2020; accepted June 20, 2020.

Author contributions: J.L.B., C.M., C.R.R., H.S., and D.J.R. designed research; J.L.B., C.M., C.R.R., C.M.E., and H.S. performed research; J.L.B., C.M., C.R.R., C.M.E., H.S., and D.J.R. analyzed data; J.L.B. edited the paper; D.J.R. wrote the paper.

${ }^{*}$ C.M.E. and H.S. contributed equally to this work.

This work was supported by National Institute on Alcohol Abuse and Alcoholism Grants R01AA012439 and R01AA026078; a Washington State University, Alcohol \& Drug Abuse Program intramural grant; and Washington State University startup funds to D.J.R. We thank Drs. llia Karatsoreos and Marina Savenkova for providing genotyped CB1R K0 mice; Breeanne Peterson for conducting additional genotyping; Dr. Beverley Rabbits for expert guidance with confocal microscopy analysis; and Dr. James Peters for discussion and guidance about experimental design, interpretation, and statistical analysis.

The authors declare no competing financial interests.

Correspondence should be addressed to David J. Rossi at david.rossi@wsu.edu.

https://doi.org/10.1523/JNEUROSCl.1931-19.2020

Copyright (@) 2020 the authors
}

the potential adverse or beneficial effects of cannabinoids on human health. This disparity is particularly stark with respect to the developing brain, wherein the role of the endocannabinoid system and modulation by exogenous cannabinoids has only been examined in a few brain subregions. Moreover, those few studies have focused primarily on cell-autonomous processes or direct interactions between cells, such as neuronal proliferation and differentiation, neuronal migration, axonal migration, and synaptogenesis, all of which are influenced by the endocannabinoid system (Trazzi et al., 2010; Díaz-Alonso et al., 2012a,b; Duff et al., 2013; Sonego et al., 2013; Maccarrone et al., 2014; Saez et al., 2014). Accordingly, where examined, application of exogenous cannabinoids disrupts these fundamental developmental processes, and presumably collectively contributes to the range of neurobehavioral impacts of in utero exposure to exogenous cannabinoids. However, one aspect of brain development that has not been widely examined is the role of the endocannabinoid system in shaping the longer-range, transcellular signaling 
cascades that coordinate neurodevelopmental processes across cells within a developing neural network (Feller, 1999; Hashimoto and Kano, 2013; Kirkby et al., 2013).

The disparity between understanding of the endocannabinoid system in brain development relative to adult function is exemplified by the cerebellum, which in the adult has one of the highest levels of expression of the dominant cannabinoid receptor CB1R (Herkenham et al., 1991; Matsuda et al., 1993; Tsou et al., 1998). Accordingly, many of the early studies that established the adult endocannabinoid system as an on-demand retrograde synaptic dampener, mediated by postsynaptic production of endocannabinoids that act on presynaptic CB1Rs to suppress vesicular transmitter release and/or induce longer-lasting forms of plasticity, were conducted at excitatory and inhibitory synapses onto cerebellar Purkinje cells (PCs) (Diana et al., 2002; Yoshida et al., 2002; Brown et al., 2003; Safo et al., 2006). Subsequent studies established that the cerebellar endocannabinoid system is crucial for motor learning (Kishimoto and Kano, 2006), and that the motor impairing effects of exogenous cannabinoid consumption are mediated by disruption of the cerebellar endocannabinoid system (DeSanty and Dar, 2001; Ramaekers et al., 2006). In contrast to our deep understanding of the subcellular distribution and mechanistic and behavioral functions of the endocannabinoid system in the adult cerebellum, very little is known about the role of this system in the developing cerebellum. This lack of information is troubling given that one of the most well-established adverse impacts of prenatal and perinatal exposure to exogenous cannabinoids in humans is impaired motor function in the offspring (Astley and Little, 1990; Willford et al., 2010; Campolongo et al., 2011). Moreover, it is now appreciated that the cerebellum also contributes to cognitive, emotional, and reward processing (Bodranghien et al., 2016), which are also affected by perinatal exposure to cannabinoids (Higuera-Matas et al., 2015), and that cerebellar malfunction is a key factor in several neurodevelopmental cognitive disorders, including attention deficit and hyperactivity disorder, autism spectrum disorder, schizophrenia, and predilection to drug/alcohol abuse (Kaplan et al., 2013; Stoodley, 2016; Hariri, 2019). Thus, improving our understanding of the role of the cerebellar endocannabinoid system during brain development, and modulation by exogenous cannabinoids is crucial to our ability to establish rational exposure guidelines for pregnant and nursing women, and to consider potential adverse interactions with or treatment options for cerebellar-related neurodevelopmental disorders that have both genetic and environmental risk factors. Here we used immunohistochemistry and brain slice patch-clamp recording to examine the expression and function of CB1R in the cerebellum of newborn rodents (postnatal day [PND] 3-12), which are neurodevelopmentally equivalent to the third trimester of gestation and early postnatal period (up to $\sim 6$ months, a key breastfeeding period) in humans (Zecevic and Rakic, 1976; Abraham et al., 2001).

\section{Materials and Methods}

Animals. All animals were bred and housed within a Washington State University vivarium, and all procedures conformed to regulations approved by the Washington State University Institutional Animal Care and Use Committee and guidelines for ethical protocols and care of experimental animals established by the National Institutes of Health. Either Sprague Dawley rats (Simonsen) or C57BL/6J transgenic mice (bred within the Washington State University vivarium; originally from Rockefeller University) were used for experiments. The C57BL/J6 CB1 KO mice were originally designed as described by Zimmer et al. (1999), in which the $C B 1$ gene was mutated by replacing its coding sequence with PGK-neo ${ }^{\mathrm{r}}$ through homologous recombination in embryonic stem cells, which completely blocks expression of the CB1 exon. All CB1 KO mice used for experiments were bred from heterozygous $C B 1^{+/-}$parents and were either homozygous $\mathrm{CB1} 1^{+/+}$(for controls) or $\mathrm{CB1}^{-/-}$.

Histology tissue sectioning. Rodent cerebelli were extracted in icecold $\left(1^{\circ} \mathrm{C}\right)$ aCSF bubbled with $95 \% \mathrm{O}_{2} / 5 \% \mathrm{CO}_{2}$ gas, and immediately placed into 4\% PFA in PBS solution, and allowed to fix for $24 \mathrm{~h}$. Tissue subsequently underwent an antifreeze preparation in which it was placed in $20 \%$ sucrose solution until sinking, followed by $30 \%$ sucrose solution ( $\sim 24 \mathrm{~h}$ process per solution). Cerebellar vermis tissue was then sectioned in the sagittal plane using a CM1950 cryostat (Leica Microsystems) at $40 \mu \mathrm{m}$ with a $-18^{\circ} \mathrm{C}$ chamber temperature. Sections were placed in a cryoprotectant solution (containing $0.1 \mathrm{M}$ sodium azide) and stored in a $-20^{\circ} \mathrm{C}$ freezer until staining.

Immunohistochemistry. Cerebellar vermal sections were taken from cryoprotectant solution and underwent a series of free-floating $1 \times$ PBS washes before a $45 \mathrm{~min}$ blocking step in 5\% BSA in 0.5\% Triton X-100/ PBS solution. Following a series of PBS washes, tissue was subjected to a 20 min incubation in solution containing nuclear stain Hoechst 33342 (8.12 mM; Invitrogen, H1399) before another series of PBS washes and finally into a $24 \mathrm{~h}$ primary antibody incubation. The tissue was then washed and placed into a $2 \mathrm{~h}$ secondary antibody incubation before a final series of PBS washes and mounted on microscope slides. The slides were then coverslipped using Permount Gold and allowed to dry until confocal imaging. All images are of slices cut in the sagittal plane.

Confocal microscopy. Fluorescence images were acquired using an SPX-8 white light point-scanning confocal microscope (Leica Microsystems) with imaging software (Leica Microsystems). Images were acquired using one of three objectives: HC PL APO $20 \times / 0.7 \mathrm{NA}$, HC PL APO CS2 $40 \times / 1.3$ NA (oil immersion), or an HC PL APO CS2 $63 \times 1$ 1.4 NA (oil immersion). For all images, laser excitation wavelengths were 488 (4\% laser), 567 (5\% laser), and $647 \mathrm{~nm}$ ( $2 \%$ laser); fluorescence emission was filtered at $522-572 \mathrm{~nm}$ for AlexaFluor-488, $602-650 \mathrm{~nm}$ for AlexaFluor-568, and $672-730 \mathrm{~nm}$ for AlexaFluor-647. Hoechst 33342 nuclear stain was excited using an ultraviolet laser, and fluorescence emission was collected at $450-500 \mathrm{~nm}$. Collection of AlexaFluor-568 images was done independently from the other scans, to reduce potential autofluorescence emission contamination from the ultraviolet laser. Digital gain and photon emission light gating (the time delay set to detect fluorescence emission only between light pulses) were identical for all species and conditions.

Acute preparation of brain slices. Rodent ages were between either PND 3-12 for third-trimester equivalent rodents or PND 30-40 for adolescent/adult (mature) rodents. Pups (PND 3-12) were housed with their dams, while mature rats (PND 30-40) were individually housed separately. All animals were kept on a standard light/dark cycle with lights on at 07:00. Cerebellar slices of randomly selected male or female rodents were prepared each day of experimentation. Rodents were anesthetized with isoflurane, and killed by decapitation. Brains were rapidly extracted into ice-cold aCSF $\left(1^{\circ} \mathrm{C}\right)$ containing the following (in $\mathrm{mm}$ ): 124 $\mathrm{NaCl}, 26 \mathrm{NaHCO}_{3}, 1 \mathrm{NaH}_{2} \mathrm{PO}_{4}, 2.5 \mathrm{KCl}, 2.5 \mathrm{CaCl}_{2}, 2 \mathrm{MgCl}_{2}$, and $10 \mathrm{D}-$ glucose. All aCSF was bubbled with $95 \% \mathrm{O}_{2} / 5 \% \mathrm{CO}_{2}$ and contained 1 mм kynurenic acid (a glutamate receptor antagonist used to prevent potential excitotoxicity within the tissue). The cerebellum was microdissected out, and parasagittal slices $(225 \mu \mathrm{m})$ of the cerebellar vermis were made using a VT1200S vibratome (Leica Microsystems) in a slicing chamber filled with bubbling $1^{\circ} \mathrm{C}$ aCSF. Slices were then incubated in aCSF containing $1 \mathrm{~mm}$ kynurenic acid at near physiological temperature $\left(34^{\circ} \mathrm{C}-35^{\circ} \mathrm{C}\right)$ for $1 \mathrm{~h}$, then in room temperature aCSF until electrophysiological recordings were conducted.

Slice electrophysiology. Slices were secured with a platinum harp in a submersion chamber mounted on an Olympus BX51WI microscope, and visualized with a $60 \times(0.90 \mathrm{NA})$ water-immersion objective. Slices were perfused at a rate of 5-7 $\mathrm{ml} / \mathrm{min}$ with aCSF, maintained at a temperature between $32^{\circ} \mathrm{C}$ and $36^{\circ} \mathrm{C}$, and bubbled with a $95 \% \mathrm{O}_{2} / 5 \% \mathrm{CO}_{2}$ gas. Granule cells (GCs), Golgi cells, stellate/basket cells, and PCs were visually identified and voltage-clamped $\left(V_{\mathrm{h}}=-30 \mathrm{mV}\right.$, except for PND 30-40 PCs, which were clamped at $-60 \mathrm{mV}$ ) with patch electrodes made 
from borosilicate glass capillary pipettes, containing the following (in mM): 130 Cs-Gluconate, $4 \mathrm{NaCl}, 0.5 \mathrm{CaCl}_{2}, 10 \mathrm{HEPES}, 5$ EGTA, 4 MgATP, $0.5 \mathrm{Na}_{2} \mathrm{GTP}$, and $5 \mathrm{QX}-314$. Internal solution was pH-adjusted to 7.2-7.3 with $\mathrm{CsOH}$. Electrode resistance was $4-8 \mathrm{M} \Omega$ for GCs and stellate/basket cells, and 1-4 $\mathrm{M} \Omega$ for Golgi and PCs. Cells were excluded if access resistance changed by $>20 \%$ across the duration of an experiment. Given the extracellular and intracellular $\left[\mathrm{Cl}^{-}\right], \mathrm{E}_{\mathrm{Cl}-}$ was $\sim-60 \mathrm{mV}$, so voltage-clamping at $-30 \mathrm{mV}$ resulted in IPSCs being outward and EPSCs being inward. All recordings were filtered at $10 \mathrm{kHz}$ and acquired at $20 \mathrm{kHz}$. In recordings from mature PCs, PCs were voltage-clamped at the $\mathrm{Cl}^{-}$reversal potential $\left(V_{\mathrm{h}}=-60 \mathrm{mV}\right)$ to isolate EPSCs, without attempting to clamp PCs at $-30 \mathrm{mV}$. A glass capillary stimulating electrode was used to evoke action potentials in local afferents, with a stimulation range of $25 \mu \mathrm{A}$ to $1.5 \mathrm{~mA}$, determined by an easily detectable and consistent amplitude evoked synaptic response. Drugs were dissolved in aCSF bubbled with $95 \% \mathrm{O}_{2} / 5 \% \mathrm{CO}_{2}$ gas before being administered. All experimental conditions were conducted for $5 \mathrm{~min}$ each, with the baseline condition being conducted for at least $5 \mathrm{~min}$, or until a stable baseline was reached.

Migrating GC ( $m G C s)$ whole-cell single channel electrophysiology. mGCs in the molecular layer (ML) were selected visually based on their small size and teardrop shape. Highly electrically compact characteristics of mGCs (i.e., small somata, no dendrites, and few channels) enabled single-channel voltage-clamp $\left(V_{\mathrm{h}}=-60 \mathrm{mV}\right)$ recordings in whole-cell configuration (Rossi and Slater, 1993). To enable recording of single NMDAR channels at $-60 \mathrm{mV}$ (required to get large enough single-channel currents to resolve in whole-cell mode), recordings were done in $\mathrm{Mg}^{2+}$-free ACSF, to relieve voltage-dependent $\mathrm{Mg}^{2+}$ block at such hyperpolarized potentials. Otherwise, all solutions were the same as used for other recordings described above. To determine whether afferent activity affected mGC NMDA channel activity, a glass capillary stimulating electrode was placed in the white matter (location of the mossy fibers [MFs]), and stimulated (paired-pulse, $20 \mathrm{~ms}$ interpulse interval) every $20 \mathrm{~s}$ at intensities ranging from 30 to $500 \mu \mathrm{A}$. Whole-cell voltage-clamp recordings were acquired and filtered as described above, and signals were further filtered at $1 \mathrm{kHz}$ for visualization and analysis of single-channel activity.

Analysis of spontaneous and evoked EPSC/IPSC currents. Spontaneous synaptic events (sIPSCs and sEPSCs) from the final 2 min of recording in each experimental condition were analyzed using Mini Analysis 6.0.7 (Synaptosoft). Automatic detection of sIPSCs and sEPSCs was executed, using an amplitude threshold of 2 times the peak-to-peak amplitude of the noise, and then events were individually inspected with a further inclusion criterion of having a rise time at least 3 times faster than the decay time. Average frequency was determined, including temporally overlapping events, then all nonoverlapping events were averaged to calculate mean amplitude. All cells with $<0.1 \mathrm{~Hz}$ spontaneous baseline frequency were excluded from analysis. Because the CB1R agonist WIN 55212-2 (WIN) fails to wash out within a reasonable amount of time, the wash condition is excluded in the following results unless noted otherwise. Electrically evoked ( $1 \mathrm{stim} / 20 \mathrm{~s} ; 10 \mathrm{stim} / 2 \mathrm{~min}$, or $1 \mathrm{stim} / \mathrm{s})$ synaptic currents were quantified using pClamp 10.4 (Clampfit; Molecular Devices). The final 10 evoked responses in every condition were averaged, and the mean peak amplitude and paired-pulse ratio was calculated.

mGC NMDAR-mediated single-channel activity analysis. The measurement of NMDAR-mediated single-channel activity in whole-cell voltage-clamped mGCs was done as previously described (Rossi and Slater, 1993) using PClamp software. Briefly, single-channel events were autodetected using a threshold of 2 times the peak-to-peak amplitude of the noise; then 100 clear "square-wave" events were selected for generation of a Gaussian to calculate the mean amplitude of these clearly resolved single-channel events. Then, because the level of NMDAR channel activity in various experiments could range from clearly resolved single channels, through harder to distinguish flurries of channel opening events, to macroscopic currents, the total current was divided by the previously determined mean single-channel amplitude, to give an estimate of the number of channels $(n)$ and the open probability $(p)$ during the last $120 \mathrm{~s}$ of a given experimental condition. Importantly, all detectable channel activity was abolished by bath application of the broad-spectrum NMDAR antagonist, D-AP5 $(50 \mu \mathrm{M})$, confirming that all forms of channel activity observed in mGCs are mediated by NMDARs.

Evoked NMDAR single-channel current analysis. To determine the total current induced by MF stimulation, first, a $1 \mathrm{~s}$ baseline region with no events was selected within a $5 \mathrm{~s}$ period immediately before the electrical stimulation. The holding current during this baseline quiescent period was subtracted from the entire trace. Then, the area of the trace $3 \mathrm{~s}$ immediately following stimulation was calculated, which provided the total evoked current $\left(\mathrm{ms}^{*} \mathrm{pA}\right)$ induced by stimulation. This response is measured as the current ( $\mathrm{pA}$ ) over this $3 \mathrm{~s}$ period, divided by singlechannel amplitude, and reported as the product of the number of channels $(n)$ and the open probability $(p)$, as described above.

Statistics. Data are expressed as the mean \pm SEM for each condition. As per Journal of Neuroscience guidelines, all figures displaying mean \pm SE also have overlaid (or displayed in parallel, if overlaying obscured easy viewing of mean values) dot plots of actual individual raw data points, and, when appropriate, have connector lines making clear the before and after outcome of a given experimental manipulation for each cell. In all experiments, statistics were performed on the number of cells used (or images used for immunocytochemistry), not the number of animals, although in every case, the number of animals from which the cells (or images) came from are indicated in the relevant figure legends. Animals of both sexes were used and were blindly distributed across experiments. For all electrophysiological experiments, each cell came from a unique slice, to avoid potential residual effects from prior drug applications. Within-cell spontaneous PSC frequencies, evoked PSC amplitudes, and mGC NMDAR channel activity before and after drug treatments were compared using paired $t$ tests. For such electrophysiological experiments, when more than one drug concentration or multiple drug or genotype conditions were compared across such conditions, ANOVA was used, with the specific type of ANOVA stated in the relevant figure legend. Nonparametric Kruskal-Wallis one-way ANOVA on Ranks were used for data groups failing a normality test (Shapiro-Wilk test). For temporally dynamic electrophysiological experiments (see Fig. 8), a two-way (see Fig. $8 C$ ) or one-way (see Fig. $8 E, G$ ) repeated-measures ANOVA with follow-up pairwise analysis (Holm-Sidak method) was used to determine overall differences, interactions, and specific data points mediating overall differences. For immunocytochemistry quantification across multiple ages (see Figs. $1 F, 2 F$ ), a Kruskal-Wallis one-way ANOVA on ranks was used. In all cases, significance was set at $p<0.05$, but exact $p$ values are provided in the legends and, when significant, on the figures as well (except when the limit of resolution was passed, $p<0.0001$ : see Figs. $1 F, 2 F, 4 L, 8 E, G)$. For statistical analysis that is not shown in figure format, the details of the statistics and exact $p$ value are in Results. A priori power analysis was not used to determine sample size, but all $n$ values are well within the range of similar types of experiments in the broader field. For all statistical tests, normality was tested with a Shapiro-Wilk test, again with $p$ value for rejection $=0.05$. All statistical measures were made using SigmaPlot 11.0 software (Systat Software) or Prism 8.0 software (GraphPad). Specific controls used throughout include using stable baseline recordings for comparison with drug responses, ensuring that drug effects are blocked by relevant antagonists or gene $\mathrm{KO}$, and replicating prior published data to ensure that novel outcomes are not because of artifactual variation across research groups (e.g., see Fig. 1B). For immunohistochemical quantification, methods were used to ensure that the person analyzing the data was blinded to the condition being analyzed (e.g., see Fig. 2D-F).

Reagents. Kynurenic acid (1 mM; Abcam, ab120064) was added to aCSF solution during brain extraction. We used $50 \mu \mathrm{M}$ AP5, $25 \mu \mathrm{M}$ NBQX, and $10 \mu \mathrm{M}$ GABAzine (Abcam, ab120003, ab120046, and ab120042 respectively) to block NMDA, AMPA/kainate, and $\mathrm{GABA}_{\mathrm{A}}$ receptors respectively. The $\mathrm{CB} 1$ agonist $(+)$-WIN was purchased from Cayman Chemical (catalog \#10009023), and CB1 inverse agonist SR141716A (Rimonabant) was purchased from Tocris Bioscience (cata$\log \#$ 0923). Primary antibodies were (host/supplier and catalog number/ dilution) as follows: CB1 (rabbit, Synaptic Systems, 258003/1:1000), Calbindin (goat, Santa Cruz Biotechnology, SC7691/1:500), and VGLUT 1/2 (chicken, Synaptic Systems, 297104/1:1000). 


\section{Results}

In the adult cerebellum, CB1R is densely expressed in the ML neuropil, in par ticular on the glutamatergic and GABAergic afferents to the sole output neuron of the cerebellar cortex, PCs (Fig. 1A,E) (Suarez et al., 2008). Activation of these CB1Rs by endogenous or exogenous agonists, including WIN, reduces vesicular release probability at these afferent terminals, which is reflected by a reduction in the frequency of miniature GABAergic and glutamatergic postsynaptic currents (Takahashi and Linden, 2000; Yamasaki et al., 2006) and the amplitude of electrically evoked eIPSCs and eEPSCs (Fig. $1 B$; eEPSC amplitude reduction by $5 \mu \mathrm{M}$ $\mathrm{WIN}=40.8 \pm 9.2 \%) \quad$ (Takahashi and Linden, 2000; Diana et al., 2002; Yoshida et al., 2002; Brown et al., 2003).

In contrast to the well-established role of $\mathrm{CB} 1 \mathrm{R}$ in the adult cerebellum, little is known about CB1R expression and function during cerebellar cortical development, which is a highly coordinated process of neuronal proliferation, differentiation, migration, and synaptogenesis that occurs during a brief time window in the early postnatal period in rodents (Fig. 1C) (Mugnaini, 1970; Okazawa et al., 2009; Leto et al., 2016; Galas et al., 2017; Kano et al., 2018). This transformation takes $\sim 3$ weeks in newborn rodents and occurs in humans from the third trimester of gestation through the early postnatal period (up to $\sim 11$ months) (Zecevic and Rakic, 1976; Abraham et al., 2001). To address this lack of information, we used confocal fluorescence imaging to map the expression pattern of CB1R in the newborn rodent cerebellum (PND 3-12; Fig. 1D-F). Similar to the adult cerebellum, immunostaining for $\mathrm{CB1R}$ is densely expressed in the expanding ML neuropil (Fig. 1D-F). However, in contrast to the adult cerebellum, there is also a dense, but punctate, expression of $\mathrm{CB} 1 \mathrm{R}$ in the developing internal GC layer (GCL; Fig. $1 D-F)$. To quantify this apparent developmental shift, we examined three key developmental time points in mice (PND 3, 9, and 22) and rats (PND 3 and 9); and to partially control for differences in antibody and/or laser light penetration through cerebellar tissue of different developmental stages, we normalized the broad CB1R staining signal intensity to the signal intensity of nuclear staining by Hoechst in the two main cellular layers (ML and GCL; Fig. $1 F$ ). In support of the raw images (Fig. $1 A, D, E$ ), such quantification revealed a significant progressive reduction in $\mathrm{CB} 1 \mathrm{R}$ staining in the GCL at each $\mathrm{PL}, \mathrm{PC}$ layer.
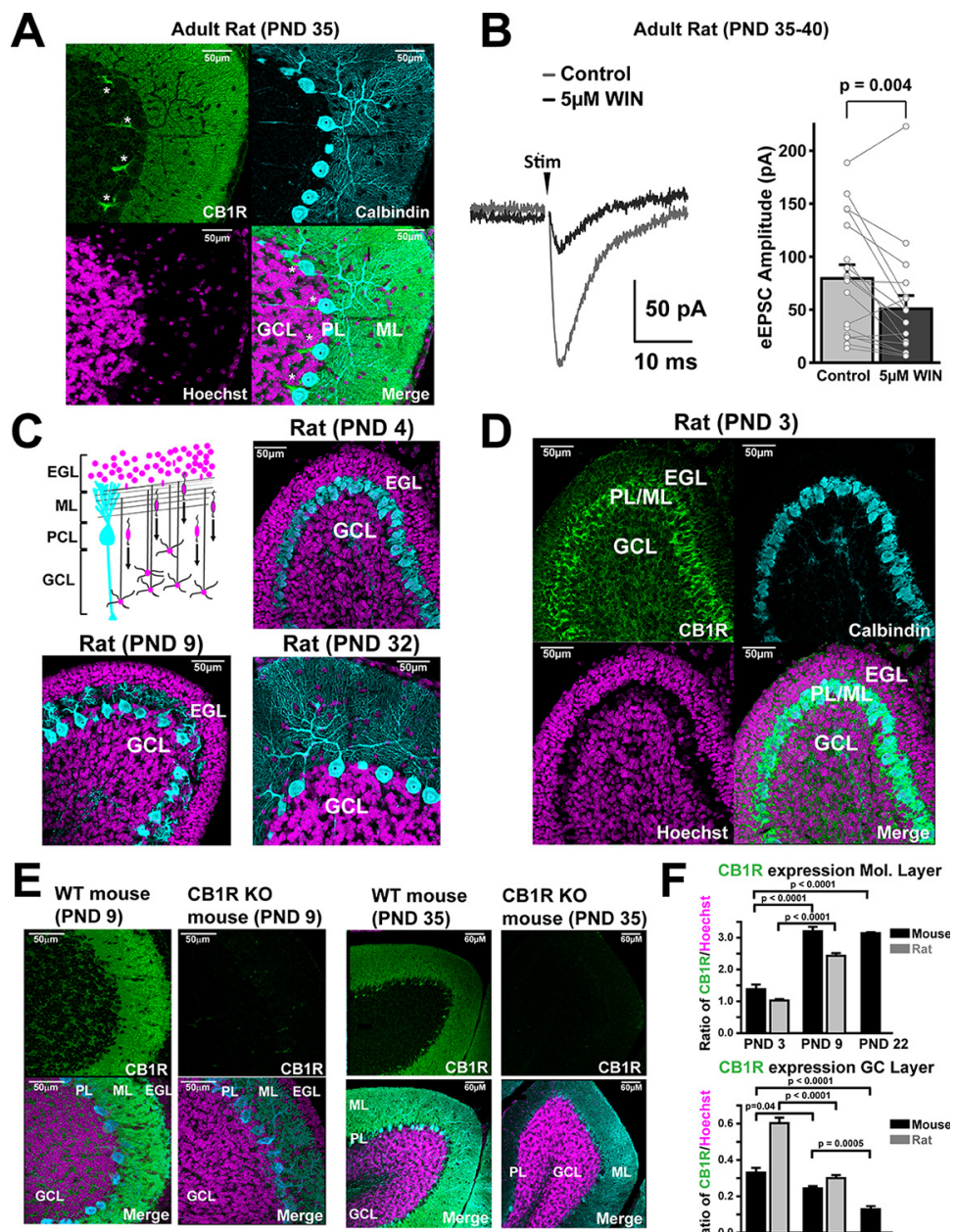

$\mathbf{F}$
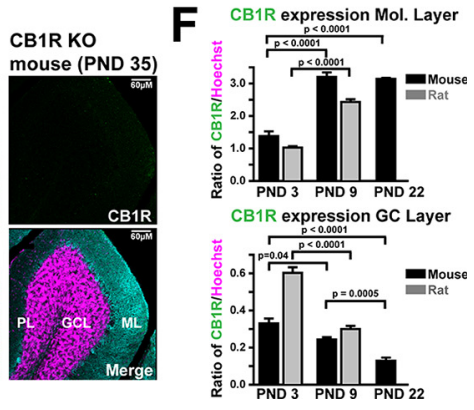

Figure 1. Developmentally transient expression of CB1Rs in the developing GCL. A, Confocally acquired images of immunohistochemistry of fully developed (PND 35) rat cerebellar cortex, showing heavy CB1R expression in the ML and on basket cell-PC synapses (*). Fluorescence emission for CB1R, Calbindin (a calcium buffering protein expressed primarily by PCS), and Hoechst (a nuclear stain) are color-coded green, cyan, and magenta, respectively. $\boldsymbol{B}$, Representative traces (left), showing that local electrical stimulation of parallel fibers evokes eEPSCs in voltage-clamped $\left(V_{h}=-60 \mathrm{mV}\right)$ PCs in cerebellar slices from PND 35-40 rats. CB1R agonist WIN $(5 \mu \mathrm{m})$ significantly reduces eEPSC amplitude (right; paired Student's $t$ test, $t_{(16)}=-3.05, p=0.004, n=16[6$ rats]). C, Schematic and confocally acquired fluorescent images represent the development of the cerebellar cortex in the early postnatal period, showing immature GCs migrating from the external granule layer (EGL) to the GCL concurrently with the proliferation of $P C$ dendritic arborization, with both processes being completed by $\sim 22$ PND (same color coding as in $A$, but without (B1R staining). D, Confocally acquired images of immunohistochemistry showing spatial expression of CB1Rs in PND 3 rat cerebellar cortex (same color coding as in $\boldsymbol{A}$ ). The main difference from adult tissue is prominent expression in the developing $\mathrm{GCL}$. $\boldsymbol{E}, \mathrm{A}$ similar CB1R staining pattern in PND 9 and PND 35 WT mouse cerebellum, but absence of signal in cerebellum from CB1R KO mice of each age confirms specificity of CB1R antibody (same color coding as in $\boldsymbol{A}$ ). $\boldsymbol{F}$, Bar graphs represent ratio of mean CB1R signal intensity over mean Hoechst signal intensity in the ML (top) and GCL (bottom) in cerebellar slices from mice (black) and rats (gray) of different postnatal ages. Top, Kruskal-Wallis one-way ANOVA on ranks $\left(\mathrm{H}_{(4)}=177.8, p<0.0001\right)$, with significant differences between pairwise comparisons by Dunn's test, with the following $z$ scores and $p$ values: rat ML PND 3 versus 9 , $z=8.4, p<0.0001$; mouse ML PND 3 versus $9, z=7.0, p<0.0001$; mouse ML PND 3 versus $22, z=5.3, p<0.0001$; mouse ML PND 9 versus $22, z=0.23, p=1.0$. Bottom, Kruskal-Wallis one-way ANOVA on ranks $\left(H_{(4)}=154.1, p<0.0001\right)$, with significant differences between pairwise comparisons by Dunn's test, with the following $z$ scores and $p$ values: rat GCL PND 3 versus 9 , $z=6.8, p<0.0001$; mouse GCL PND 3 versus $9, z=2.9, p=0.04$; mouse GCL PND 3 versus $22, z=5.6, p<0.0001$; mouse $\mathrm{GCL}$ PND 9 versus $22, z=4.1, p=0.0005$. Values are derived from $n=29-111$ images, from at least 4 animals at each age/species).

developmental stage (Fig. $1 F$, bottom). This developmental reduction in the GCL was paralleled by a significant increase in expression levels in the ML from PND 3 to PND 9, after which expression levels were stable (Fig. $1 F$, top). The observed immunostaining pattern and changes across development are specific to $\mathrm{CB} 1 \mathrm{R}$ expression because staining is absent in developing and adult brain tissue from mice with germline deletion of $\mathrm{CB} 1 \mathrm{R}$ (Fig. 1E). 

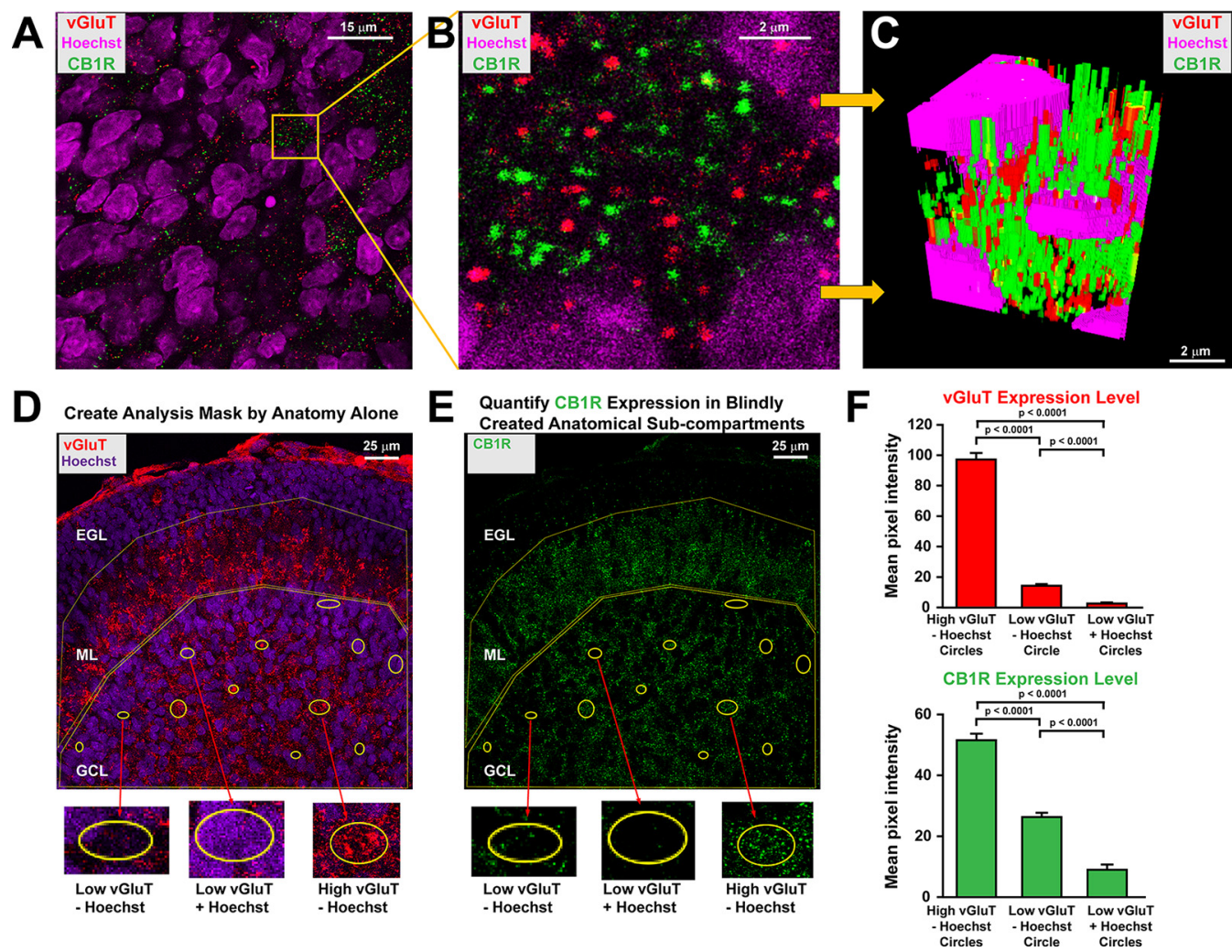

Figure 2. Developmentally transient expression of $\mathrm{CB} 1 \mathrm{Rs}$ in developing $\mathrm{GCL}$ is concentrated on afferent MF terminals. $A, B$, Confocally acquired fluorescent images of immunohistochemistry of developing (PND 3) rat cerebellar cortex, showing subcellular distribution of CB1R expression in the developing GCL. Fluorescence emission for CB1R, vGluT1 and vGlut2 (the vesicular glutamate transporters located in glutamatergic MF terminals), and Hoechst (a nuclear stain) are color-coded green, red, and magenta, respectively. $\boldsymbol{B}$, Magnified view of the boxed region in $\boldsymbol{A}$. $\boldsymbol{C}$, 3D projection $(10 \mu \mathrm{m} \times 10 \mu \mathrm{m} \times 10 \mu \mathrm{m})$ of the image stack shown in $2 D$ in $\boldsymbol{B}$ shows colocalization of vGluT and CB1R in nuclear-free volumes of $\sim 15-25 \mu \mathrm{m}^{3}$. $\boldsymbol{D}$, Example of Hoechst and vGluT only images that were used to define subcellular compartments: (1) high vGluT and no Hoechst [presumed glutamatergic MF terminals], (2) low vGluT with Hoechst [presumed neuronal and astrocytic somas], and (3) low vGluT, no Hoechst [presumed neuronal and astrocytic processes]. Circles overlaid on image are examples of so defined ROls (representative examples shown at higher magnification in bottom panels). $\boldsymbol{E}$, The (B1R channel from the same image in $\boldsymbol{D}$, showing (B1R expression in the blindly defined subcellular regions (circles). $\boldsymbol{F}$, Quantification of mean vGluT (top) and CB1R (bottom) fluorescence intensity in the three subcellular compartments. CB1R expression (bottom) is significantly concentrated in presumed afferent MF terminals, defined as nucleus lacking compartments with high vGluT expression (as subjectively identified in $\boldsymbol{D}$ and quantitatively defined in $\boldsymbol{F}$, top). Top, Kruskal-Wallis one-way ANOVA on ranks $\left(\boldsymbol{H}_{(2)}=\right.$ 213.9, $p<0.0001$ ), and significant differences between pairwise comparisons by Dunn's test with the following $z$ scores and $p$ values: vGluT in vGluT high versus vGluT low circles, $z=7.7$, $p<0.0001$; vGluT in vGluT high versus Hoechst circles, $z=14.6, p<0.0001$; vGluT in vGluT low versus Hoechst circles, $z=6.9, p<0.0001$. Bottom, Kruskal-Wallis one-way ANOVA on ranks $\left(H_{(2)}=160.6, p<0.0001\right)$, and significant differences between pairwise comparisons by Dunn's test with the following $z$ scores and $p$ values: CB1R in vGluT high versus vGluT low circles, $z=5.4, p<0.0001$; CB1R in vGluT high versus Hoechst circles, $z=12.6, p<0.0001$; CB1R in vGluT low versus Hoechst circles, $z=7.2, p<0.0001$. Values are derived from $n=84$ images, from 5 rats.

To gain insight into the subcellular localization of the developmentally transient expression of CB1R within the GCL, we conducted higher-magnification confocal studies of CB1R expression, combined with immunostaining for a marker of afferent MF terminals, the vesicular glutamate transporters, vGluT1 and 2 (Fig. 2). Notably, although expressed throughout the GCL, CB1R staining appeared to be concentrated in regions lacking nuclei and with high levels of signal for vGluT (Fig. 2AC). Importantly, as seems evident from $2 \mathrm{D}$ images (Fig. $2 A, B$ ), $3 \mathrm{D}$ projections confirmed that this consistent colocalization of CB1R and vGluT in nuclear-free regions is not at a molecular level (i.e., there is not much color overlap), but rather the two epitopes co-occupy 3D nuclear-free volumes with diameters on the order of 2-5 $\mu \mathrm{m}$ (Fig. $2 \mathrm{C}$ ). MF afferent terminals are the only known subcellular structure within the GCL with those dimensions, which are both nucleus-free and have clear expression of vGluT. To quantify this apparent expression of CB1R in MF afferent terminals, we identified subcellular compartments with the analyst blinded to the expression of CB1R (Fig. 2D-F). In particular, we subjectively identified three subregions (Fig. 2D): (1) those lacking nuclei, but with high vGluT expression (presumed MF afferent terminals); (2) those with nuclei, which always had low vGluT expression (presumed to be GC, Golgi cell, or astrocyte cell bodies); and (3) those lacking nuclei but also having low expression of vGluT (presumed to be axons, dendrites, or astrocyte processes). Quantification of mean vGluT expression intensity within the blindly identified subcellular compartments confirmed significantly disparate expression levels of vGluT in respective compartments (Fig. $2 F$, top). Subsequent quantification of CB1R expression within the respective subcellular compartments confirmed that CB1R is concentrated in presumed MF afferents, with significantly higher levels of CB1R expression in high vGluT, nucleus-free subregions compared with the nonafferent compartments (Fig. 2F, bottom). Collectively, our immunohistochemical studies indicate that the developmentally transient expression of CB1R within the GCL is concentrated in MF afferents.

To determine the function of CB1Rs in the respective locations, we conducted voltage-clamp recordings of synaptic currents in PCs (Fig. 3), ML and GCL interneurons (Fig. 4), and GCs (Fig. 5) in acutely prepared slices of cerebellum. Voltage-clamped $\left(V_{\mathrm{h}}=-30 \mathrm{mV}\right)$ PCs exhibited sEPSCs and sIPSCs (mean 
frequency $=0.72 \pm 0.16$ and $1.28 \pm$ $0.29 \mathrm{~Hz}$, respectively; Fig. 3B). Surprisingly, bath application of WIN (5 $\mu \mathrm{M})$ did not significantly affect the frequency or amplitude of PC sEPSCs or sIPSCs (Fig. 3B; frequency $\%$ change $=$ $11.0 \pm 17.3$ and $-15.7 \pm 8.1$, respectively; amplitude \% change $=2.9 \pm 4.3$ and $-6.6 \pm 3.5$, respectively). WIN also failed to significantly affect the amplitude of eEPSCs and eIPSCs elicited by local electrical stimulation of the neuropil (Fig. 3A,C,D; \% change = $19.0 \pm 10.6$ and $-3.5 \pm 13.1$, respectively). Similarly, WIN did not affect eEPSC or eIPSC decay kinetics (data not shown; WIN-induced \% change in $10 \%-90 \%$ decay time for eEPSC and IPSCs, respectively $=9.01 \pm 0.98, n=$ 11, Wilcoxon Signed Rank test, $z=$ $-0.087, p=0.27$ and $8.19 \pm 10.19$, $n=4$, paired Student's $t$ test, $t_{(3)}=$ $-0.72, p=0.52$ ), arguing against a potential presynaptic action that was simply not detected by saturated postsynaptic receptors. Thus, despite similar gross localization within the ML, activation of CB1Rs does not affect synaptic transmission from parallel fibers or interneurons to PCs.

Given the lack of effect of WIN on synaptic currents onto PCs (Fig. 3) despite dense CB1R expression in the developing ML (Fig. 1D-F), we conducted voltage-clamp recordings from ML interneurons (stellate and basket cells) and GCL Golgi cells, all of which also have their dendritic trees in the ML neuropil (Fig. 4). Stellate and basket cells were identified by their location and process arborization within the ML (Fig. 4A,B). Although some ML interneurons exhibited classic stellate or basket cell location and morphology (Fig. 4B), given the difficulty of definitively discriminating between the two subtypes of interneuron, especially at this developmental age, electrophysiological data obtained from the respective cell types were pooled. Golgi cells were identified by the location of their soma within the GCL, combined with a larger soma size, far more extensive process arborization (Fig. $4 E, F)$ and their associated larger capacitance compared with GCs (mean area of capacitive transient $=413.2 \pm 53.7$ and $75.0 \pm 9.6 \mathrm{pA}^{*} \mathrm{~ms}$ for Golgi and GCs, respectively; Fig. $4 F$ ). In voltage-clamp recordings $\left(V_{\mathrm{h}}=-30 \mathrm{mV}\right)$, all three cell types exhibited sIPSCs and sEPSCs (mean sIPSC and sEPSC frequency, respectively = $0.96 \pm 0.31$ and $0.92 \pm 0.20$ for stellate/basket cells, Fig. 4C,D; and $0.39 \pm 0.16$ and $0.58 \pm 0.23$ for Golgi cells; Fig. $4 G, H$ ), which were abolished by bath application of respective $\mathrm{GABA}_{\mathrm{A}}$ and glutamate receptor antagonists, GABAzine $(10 \mu \mathrm{M})$ and AP5 $(50 \mu \mathrm{M})+$ NBQX $(25 \mu \mathrm{M})$ (Fig. $4 C, D$ and $4 G, H$, respectively). Bath application of WIN significantly suppressed the frequency of sIPSCs in all three cell types (\% reduction for stellate/basket
C PC eEPSC
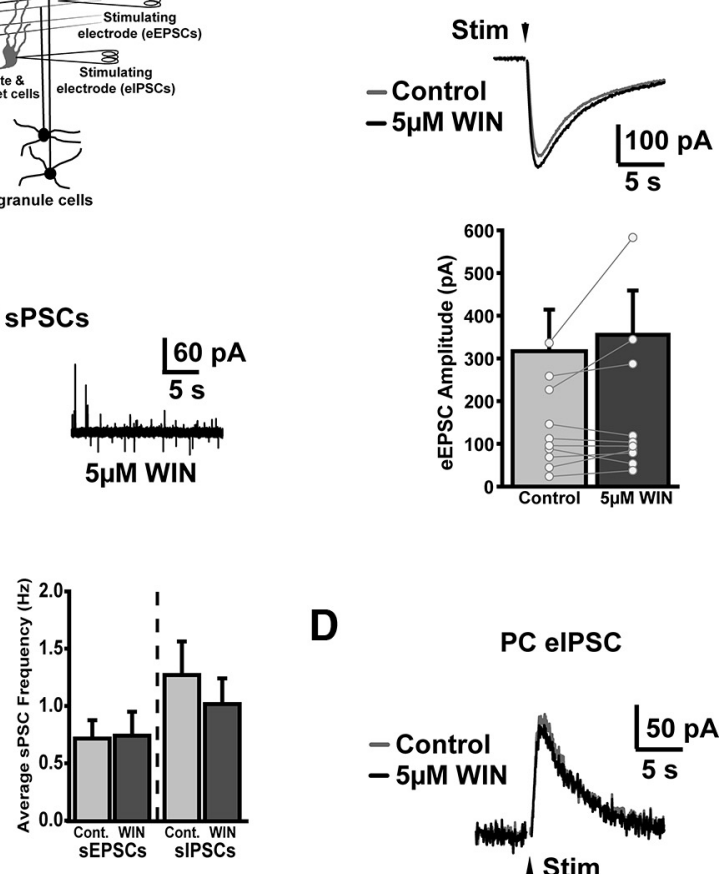

D PC eIPSC
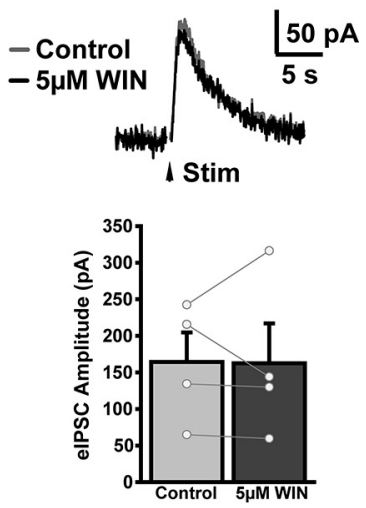

Figure 3. Activation of CB1Rs does not affect spontaneous or evoked EPSCs or IPSCS in PCS of newborn rats. A, Circuit diagram GABAergic stellate/basket cell PC afferents. $\boldsymbol{B}$, Representative traces (top), and quantification of sEPSC and sIPSC frequency (middle) and amplitude (bottom) in voltage-clamped PCs $\left(V_{\mathrm{h}}=-60 \mathrm{mV}\right)$ in slices of cerebellum from newborn rats (PND 4-14). WIN Student's $t$ test, $t_{(16)}=1.14, p=0.27$, respectively) or amplitudes (paired Student's $t$ test, $t_{(19)}=-0.98, p=0.31$ and paired Student's $t$ test, $t_{(19)}=1.38, p=0.19$, respectively), $n=29$ [8 rats]. $\boldsymbol{C}, \boldsymbol{D}$, WIN $(5 \mu \mathrm{m})$ does not significantly affect PC eEPSC am(C) Wilcoxon Signed Rank test, $z=-1.5, p=0.15, n=13$ [4 rats]) or elPSC amplitude (D; paired Student's $t$ test, $t_{(3)}=$ $0.06, p=0.95, n=4$ [1 rat]). PC EPSCS and IPSCs were evoked by local stimulation of glutamatergic parallel fibers and GABAergic stellate and basket cells, respectively (as depicted in $\boldsymbol{A}$ ).

cells $=28.3 \pm 10.3$, Fig. $4 I, J$; and for Golgi cells $=45.14 \pm 7.71$, Fig. $4 K, L)$. In contrast, WIN suppressed the frequency of sEPSCs only in Golgi cells ( $\%$ reduction $=36.97 \pm 6.54$; Fig. $4 I-L$ ). These data indicate that the dense expression of CB1R in the ML neuropil reflects functional presynaptic CB1Rs on inhibitory interneuron collaterals onto all subtypes of inhibitory interneuron and on parallel fibers, but restricted to sites that affect synapses with Golgi cells, but not PCs, stellate cells, or basket cells.

To ascertain the function of the punctate expression of CB1Rs in the developing GCL, and its apparent expression on MF terminals (Fig. 2), we next examined excitatory synaptic currents in GCs (Fig. 5). Voltage-clamped $\left(V_{\mathrm{h}}=-30 \mathrm{mV}\right)$ GCs exhibited sEPSCs (mean frequency $=0.59 \pm 0.21 \mathrm{~Hz}$; Fig. $5 A, B$ ) and robust eEPSCs elicited by electrical stimulation of the white matter MFs (amplitude $=61.3 \pm 11.0 \mathrm{pA}$; Fig. $5 E$ ). GCs also exhibited sIPSCs, which were not analyzed for this study. In contrast to the lack of action in PCs, WIN significantly reduced the 
A
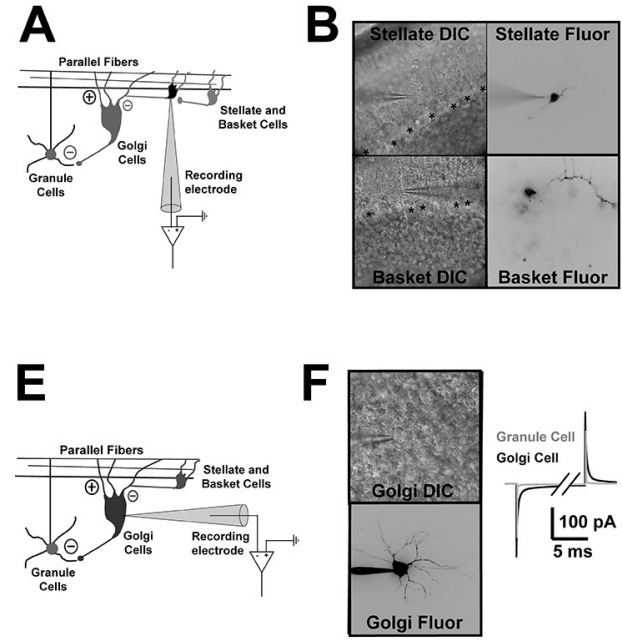
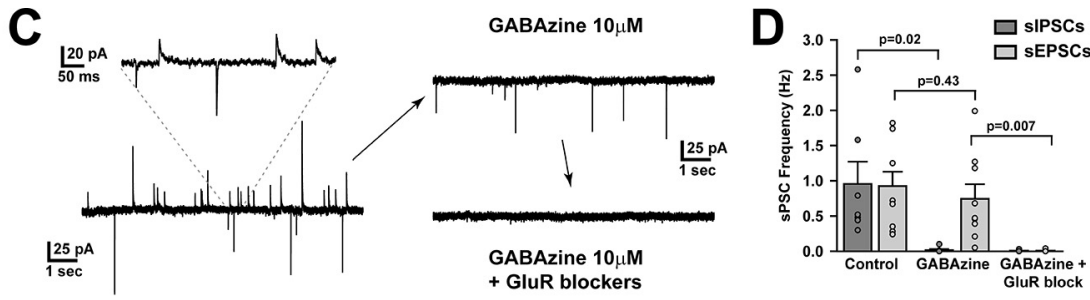

+ GluR blockers
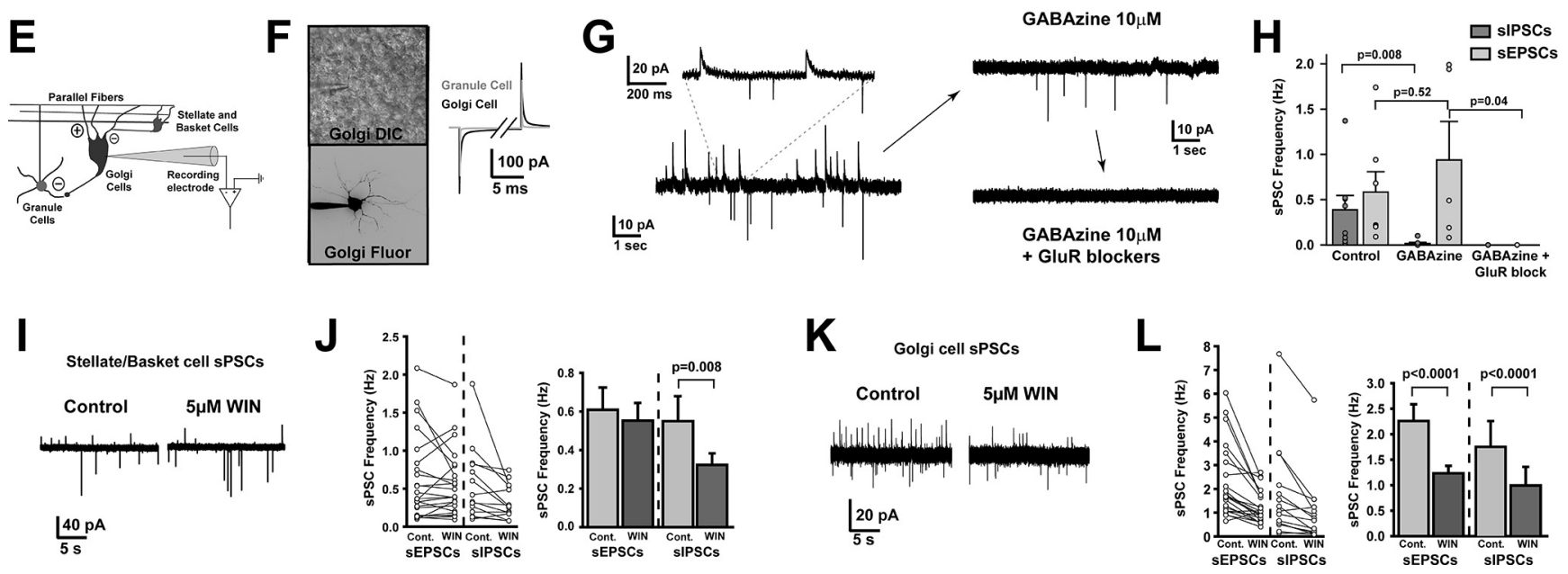

Figure 4. CB1Rs exhibit a developmentally unique pattern of synaptic suppression at ML interneuron synapses in newborn rats. $A, B$, Circuit diagram $(\boldsymbol{A})$ and DIC/fluorescence images $(\boldsymbol{B})$ of location and morphology used to identify stellate/basket cell recordings (for orientation, ${ }^{* \prime} \mathbf{s}$ in $\boldsymbol{B}$ mark individual Purkinje cells in the $\mathbf{P C}$ layer). $\boldsymbol{C}, \boldsymbol{D}$, Representative traces $(\boldsymbol{C})$ and quantification of sEPSCs and sIPSCS $(\boldsymbol{D})$ in voltage-clamped $\left(V_{\mathrm{h}}=-30 \mathrm{mV}\right)$ stellate/basket cells showing that they are, respectively, blocked by the glutamate receptor antagonists (50 $\mu \mathrm{m}$ AP5 and $25 \mu \mathrm{m}$ $\mathrm{NBQX})$ and GABA ${ }_{A} R$ antagonists, GABAzine $(10 \mu \mathrm{m})$. Wilcoxon Signed Rank Test, $z=-2.4, p=0.02$ and $z=-0.89, p=0.43$ for GABAzine effects on sIPSC and sEPSC frequencies, respectively, and Student's $t$ test, $t_{(8)}=3.6, p=0.007$, for AP5/NBQX effects on SEPSC frequency. $\boldsymbol{E}, \boldsymbol{F}$, Circuit diagram $(\boldsymbol{E})$ and DIC/fluorescence images of location and morphology ( $\boldsymbol{F}$, left) with representative capacitive transients for Golgi cell (black trace) and GCS (gray trace; $\boldsymbol{F}$, right) used to identify Golgi cells within the GCL. $\boldsymbol{G}, \boldsymbol{H}$, Representative traces (G) and quantification of sEPSCs and sIPSCS $(\boldsymbol{H})$ in voltage-clamped $\left(V_{\mathrm{h}}=-30 \mathrm{mV}\right)$ Golgi cells, showing that they are, respectively, blocked by the glutamate receptor antagonists (50 $\mu \mathrm{m} A P 5$ and $\left.25 \mu \mathrm{m} N B Q X\right)$ and GABA $R$ antagonists, GABAzine $(10 \mu \mathrm{m})$. Wilcoxon Signed Rank Test, $z=-2.52, p=0.008$ and Student's $t$ test, $t_{(4)}=-0.71, p=0.52$ for GABAzine effects on sIPSC and sEPSC frequencies, respectively, and Student's $t$ test, $t_{(4)}=3.6, p=0.04$, for AP5/NBQX effects on sEPSC frequency. I, J, WIN $(5 \mu \mathrm{m}$ ) significantly reduces the frequency of stellate/basket cell sIPSCs (Wilcoxon Signed Rank test, $z=-2.6, p=0.008, n=14$ [6 rats]) without affecting the frequency of sEPSCs (Wilcoxon Signed Rank test, $z=-0.4, p=0.43, n=24$ [7 rats]) in slices from PND 5-10 rats. $K, \boldsymbol{L}$, WIN $(5 \mu \mathrm{m})$ significantly reduces the frequency of both sEPSCS (Wilcoxon Signed Rank test, $z=-3.8, p<0.0001, n=22[6$ rats]) and sIPSCs (Wilcoxon Signed Rank test, $z=-3.2, p<0.0001$, $n=15$ [5 rats]) in Golgi cells in slices from PND 8-10 rats.

frequency of GC sEPSCs (\% reduction $=63.8 \pm 7.7 ;$ Fig. $5 B)$ and the amplitude of eEPSCs (\% reduction $=38.7 \pm 7.8$; Fig. $5 E$ ), effects that did not readily recover on return to control aCSF (data not shown). Both effects were prevented by the CB1R antagonist SR14716A (SR; Fig. 5C,G). SR did not significantly affect basal sEPSC frequency (paired Student's $t$ test, $t_{(8)}=1.14$, $p=0.29$ and $t_{(7)}=1.07, p=0.30$ for 1 and $2 \mu \mathrm{M}$ respectively, and Wilcoxon Signed Rank, $z=-0.67, p=0.63$ for $8 \mu \mathrm{M}$ ) or eEPSC amplitude (paired Student's $t$ test, $t_{(9)}=-0.51, p=0.62$ ) before application of WIN (data not shown). Actions of WIN were also abolished by germline genetic deletion of CB1R (Fig. 5D), confirming mediation by CB1Rs. Suppression of eEPSC amplitude was associated with an increase in the paired-pulse ratio (\% increase $=49.1 \pm 16.14$; Fig. $5 F$ ), supporting a presynaptic locus of action compatible with immunostaining for CB1R on MF terminals (Fig. 2). To determine whether the observed CB1R expression and function at MF to GC synapses were simply a hitherto unnoticed general process across all ages versus a transient developmental process, we made voltage-clamp $\left(V_{\mathrm{h}}=\right.$ $-30 \mathrm{mV}$ ) recordings from GCs in slices of cerebellum from adult rats (PND 35-40). In such recordings, WIN did not affect GC sEPSC frequency (Fig. $5 H$ ), demonstrating that both expression and function at MF to GC synapses are temporally restricted to the growth spurt of cerebellar development.
Collectively, the data indicate that CB1Rs in the developing cerebellum function similarly to what has been reported in adult brain (i.e., they reduce vesicular release probability); thus, the strength of synaptic transmission. However, CB1Rs exhibit a developmentally unique expression pattern, such that CB1Rinduced suppression of synaptic transmission occurs at afferent inputs to GCs and at local inhibitory neuron synapses, instead of at synapses onto efferent output PCs that dominate in the adult cerebellum.

While the transient expression of CB1Rs on cerebellar afferents could simply serve a local synaptic developmental function (Okazawa et al., 2009; Dhar et al., 2018), their suppression of GC synaptic excitation could also affect downstream transmission, which plays important roles in coordinating the diverse and spatially dispersed cerebellar developmental processes (Fig. 1C) that must be temporally orchestrated if appropriate network connectivity is to be achieved (Kano et al., 2018; Schilling, 2018). One well-established trans-network, developmentally coordinated process is PC climbing fiber pruning, which, despite being a separate afferent system, is dependent on appropriate GC activity during critical developmental periods (Kano et al., 2018). Since CB1Rs do not affect monosynaptic transmission to PCs (Fig. 3), we tested whether actions at the MF to GC synapse affect downstream PC excitation. To do this, we made voltage-clamp 
A

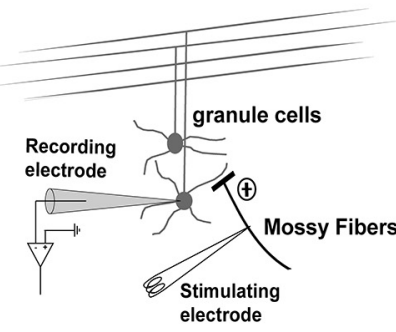

B Rat GC (PND 4) SEPSCS

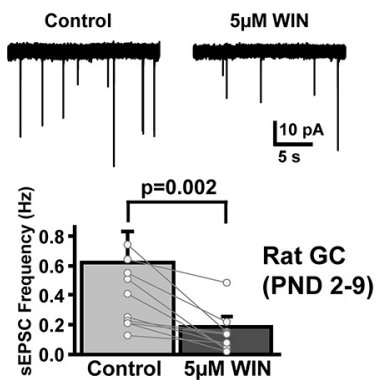

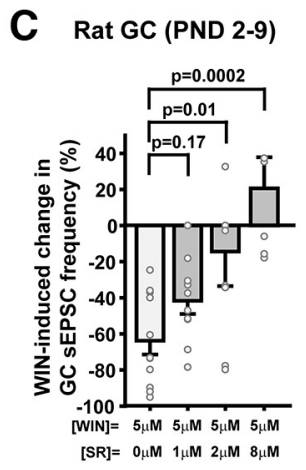

D
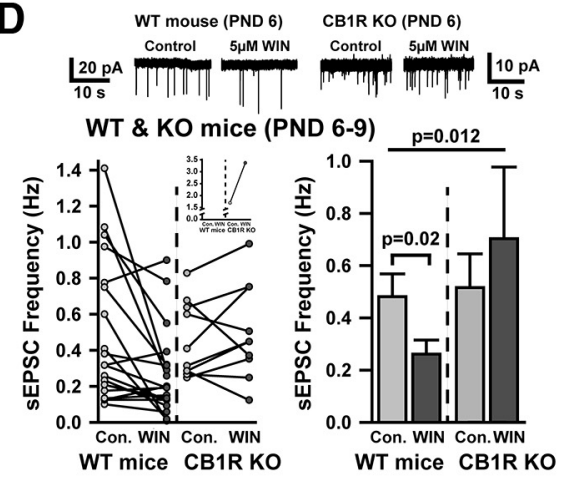

E

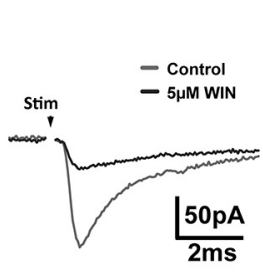

$\mathbf{F}$

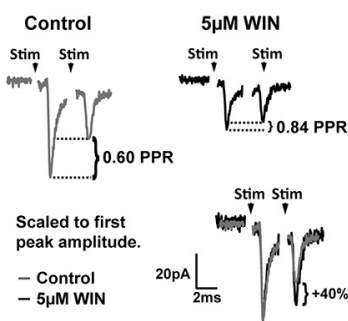

Rat GC (PND 2-12) eEPSCs

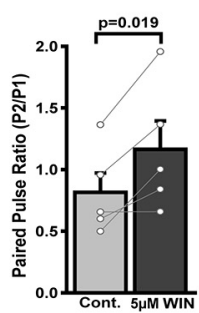

G Rat GC (PND 2-12)
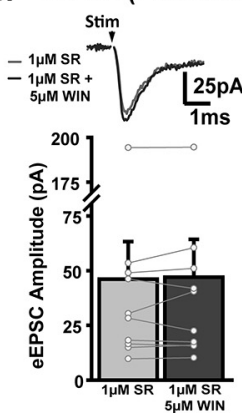

H Rat GC (PND 35-40)
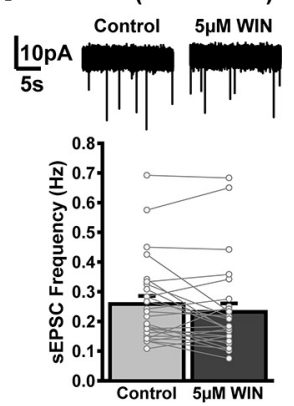

Figure 5. Activation of CB1Rs suppresses MF afferent excitation of GCs in newborn rats but not adult rats. $A$, Circuit diagram showing whole-cell patch recording of GCs and location of stimulating electrode at glutamatergic MF afferents. $B$, Representative traces (top) and quantification of sEPSCS (bottom) in voltage-clamped $\left(V_{\mathrm{h}}=-30 \mathrm{mV}\right) \mathrm{GCS}$, showing that WIN $(5 \mu \mathrm{M})$ significantly reduces the frequency of $\mathrm{SEPSCS}$ (paired Student's $t$ test, $t_{(9)}=4.3, p=0.002, n=10[5$ rats]) in slices from PND 2-9 rats. C, Bar chart showing that the (B1R antagonist, SR141716A, dose-dependently blocks the WIN-induced suppression of sEPSC frequency (one-way ANOVA, $F_{(3,31)}=7.89, p=0.0005$, Holm-Sidak multiple comparisons to WIN alone: $1 \mu \mathrm{m}$ SR, $t_{(31)}=1.40$, $p=0.17,2 \mu \mathrm{m} \mathrm{SR}, t_{(31)}=2.93, p=0.01,8 \mu \mathrm{m} \mathrm{SR}, t_{(31)}=4.59, p=0.0002$ ). $\boldsymbol{D}$, Representative traces (top panels) and quantification of sEPSC frequency (bottom panels) in voltage-clamped $\left(V_{\mathrm{h}}=-30 \mathrm{mV}\right) \mathrm{GCS}$, showing that WIN $(5 \mu \mathrm{m})$ significantly reduces the frequency of sEPSCs in PND 6-9 WT mice but not in CB1R KO mice $(2 \times 2$ ANOVA, strain $\times$ treatment interaction, $F_{(1,29)}=7.23, p=0.012$, strain main effect, $F_{(1,29)}=1.99, p=0.17$, treatment main effect, $F_{(1,29)}=0.05, p=0.831$, Holm-Sidak pairwise comparisons Cont. versus WIN within WT, $t_{(18)}=$ $2.44, p=0.02$, Cont. versus WIN within $\left.\mathrm{KO}, t_{(9)}=1.54, p=0.13\right)$. $E$, Representative traces (left) and quantification of MF-evoked GC EPSC amplitudes (eEPSCS), showing that WIN (5 $\left.\mu \mathrm{M}\right)$ significantly reduces the amplitude of eEPSCs (Wilcoxon Signed Rank test, $z=3.05, p=0.001, n=16$ [6 rats]). $\boldsymbol{F}$, Representative traces (left panels) and quantification of MF-evoked paired-pulse stimulation (ISI $=20 \mathrm{~ms})$ EPSC amplitudes, showing that WIN $(5 \mu \mathrm{M})$ suppression of eEPSC amplitude is accompanied by a significant increase in paired-pulse ratio (paired Student's $t$ test, $t_{(4)}$ $=-3.8, p=0.019, n=5$ [2 rats]). Scale bars are for isolated traces only; interstimulus recording has been removed for clarity. $\mathbf{G}$, Representative traces (top) and quantification of MF-evoked EPSC amplitudes, showing that WIN $(5 \mu \mathrm{m})$ suppression of eEPSC amplitude is prevented by the CB1R antagonist, SR141716A (1 $\mu \mathrm{m}$; paired Student's $t$ test, $t_{(9)}=0.6, p=0.55, n=8$ [4 rats]). $\boldsymbol{H}$, Representative traces (top) and quantification of GC SEPSC frequency, showing that WIN $(5 \mu \mathrm{m})$ does not significantly affect sEPSC frequency in PND 35-40 rats (paired Student's $t$ test $t_{(26)}=1.9, p=0.07, n=27[12$ rats $\left.]\right)$.

recordings $\left(V_{\mathrm{h}}=-30 \mathrm{mV}\right)$ of $\mathrm{PC}$ polysynaptic EPSCs evoked by electrical stimulation of GC afferent MFs (Fig. 6). MF stimulation $(75 \mu \mathrm{A}$ to $1 \mathrm{~mA})$ reliably elicited polysynaptic EPSCs (mean amplitude $=280.2 \pm 69.9 \mathrm{pA}$; Fig. $6 A, B)$. Bath application of WIN $(5 \mu \mathrm{M})$ significantly suppressed the magnitude of the polysynaptic EPSC (\% reduction $=45.5 \pm 6.5$; Fig. $6 B, C$ ). Such suppression of MF-evoked PC polysynaptic EPSCs was abolished by the CB1R antagonist SR14716A (SR; $2 \mu \mathrm{m}$; Fig. 6D). Thus, while CB1Rs do not affect synaptic transmission at monosynaptic synapses onto PCs, activation of CB1Rs on MF to GC synapses suppresses transmission of afferent synaptic signals through GCs to downstream PCs.

Another key cerebellar developmental process during the postnatal period is the terminal differentiation of GCs in the external GCL, followed by their migration across the developing ML to their adult location in the mature GCL (Fig. 1C). In rodents, the success of this process requires that $\sim 100$ million GCs terminally differentiate, migrate $\sim 200-300 \mu \mathrm{m}$, and form appropriate excitatory and inhibitory synapses with incoming MFs and Golgi afferents, respectively, all within a 3 week period. While the mechanistic details of this enormous task are not fully understood, it is well established that GC migration rate is driven by $\mathrm{Ca}^{2+}$ influx through single, nonsynaptic glutamate receptor- gated channels of the NMDAR subtype (Komuro and Rakic, 1993; Rossi and Slater, 1993). The level of NMDAR channel activity increases during development, in part via increased NMDAR channel expression density (Rossi and Slater, 1993), potentially to enable more rapid migration rate across an increasingly greater migrational distance. In addition to adjusting migration rate to match distance needed to be traversed, it is likely, albeit untested, that migration rate should be adjusted such that GC arrival coincides with the arrival and maturation of their eventual synaptic afferents. If such coordination exists, then changes in GC afferent excitation should affect the NMDAR channel activity that drives GC migration rate. To test this hypothesis, we made patch-clamp recordings from mGCs in the ML, identified by their location, size, and morphology (Fig. 7). As we reported previously, in zero $\mathrm{Mg}^{2+}$ aCSF (to eliminate voltage-dependent $\mathrm{Mg}^{2+}$ block of NMDAR channels) (Mayer et al., 1984) at the hyperpolarized potentials required to resolve singlechannel events in whole-cell mode (Rossi and Slater, 1993; Ebralidze et al., 1996), voltage-clamped $\left(V_{\mathrm{h}}=-60 \mathrm{mV}\right) \mathrm{mGCs}$ exhibit spontaneous NMDAR channel openings (mean singlechannel current $=-5.1 \pm 0.1 \mathrm{pA}$; Fig. $7 C, D$ ) that are abolished by the NMDAR receptor antagonist, AP5 (50 $\mu$; Fig. $7 C, D$ ). Although such spontaneous NMDAR channel activity is caused 
A

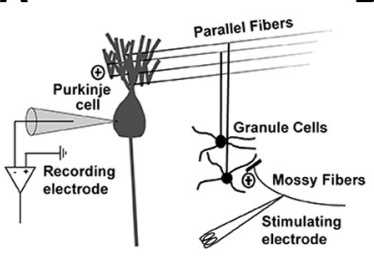

B

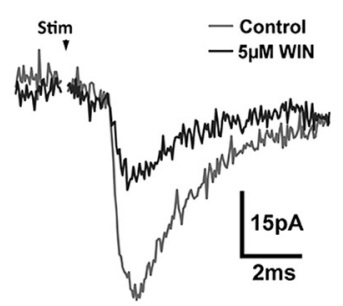

C

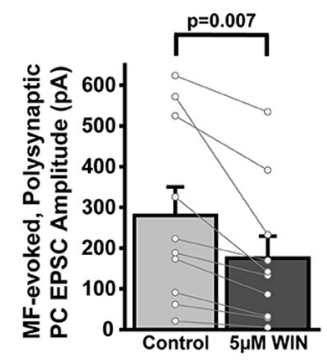

D

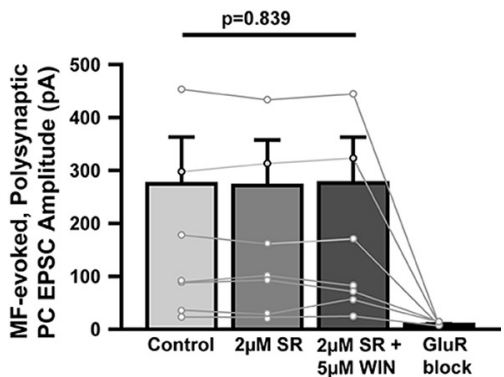

Figure 6. Activation of CB1Rs suppresses MF afferent-evoked polysynaptic excitation of PCs in newborn rats. $A$, Circuit diagram showing whole-cell patch recording of $P C S$ and location of stimulating electrode at glutamatergic MF afferents. $\boldsymbol{B}-\boldsymbol{D}$, Representative traces $(\boldsymbol{B})$ and quantification of MF-evoked, polysynaptic EPSCS $(\boldsymbol{C}, \boldsymbol{D})$ in voltage-clamped $\left(V_{\mathrm{h}}=-30 \mathrm{mV}\right)$ PND $5-8$ PCS, showing that WIN $(5 \mu \mathrm{M})$ significantly reduces the amplitude of MF-evoked polysynaptic PC EPSCS (C; Wilcoxon Signed Rank test, $z=2.8, p=0.002, n=10[5$ rats]), and (D) that the CB1R antagonist SR $(2 \mu \mathrm{m})$ does not affect basal MF-evoked polysynaptic PC EPSC amplitude but prevents subsequent suppression by WIN $\left(5 \mu \mathrm{m} ;\right.$ one-way repeated-measures ANOVA, $F_{(2)}=0.2$, $p=0.839, n=7[4$ rats] ). MF-evoked polysynaptic responses were blocked by glutamate receptor antagonists AP5 (50 $\mu \mathrm{m})$ and NBQX (25 $\mu \mathrm{m})$. Far right bar, GluR block.

A

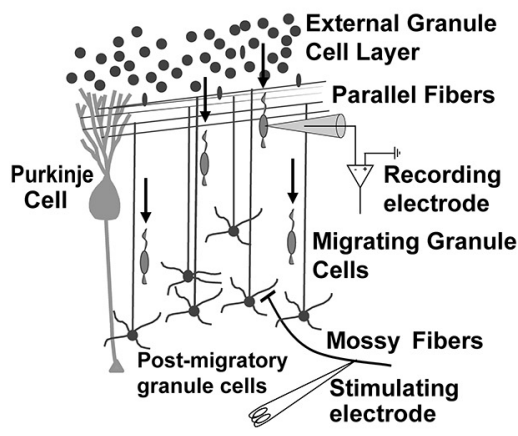

C

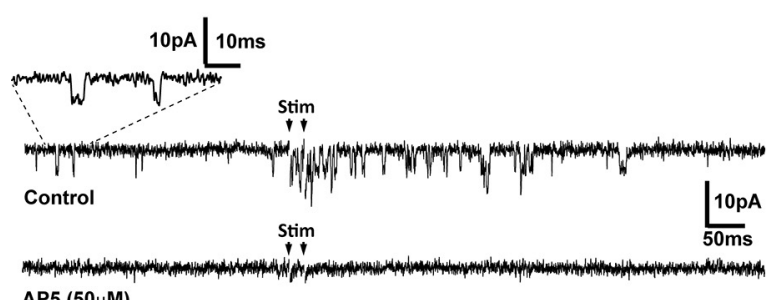

B

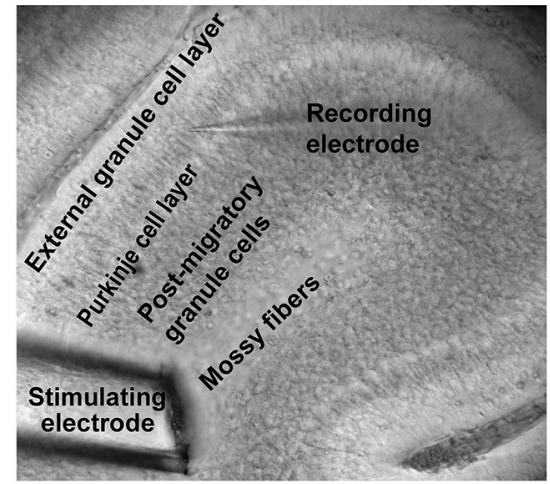

D

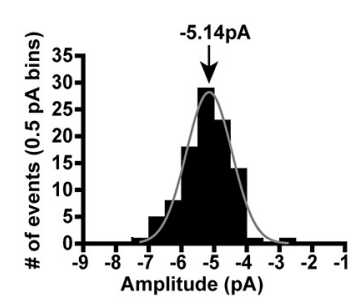

E

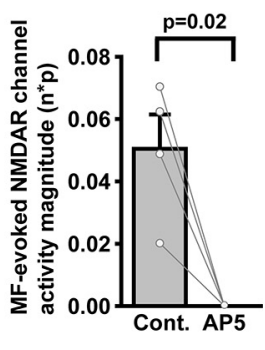

$\mathbf{F}$

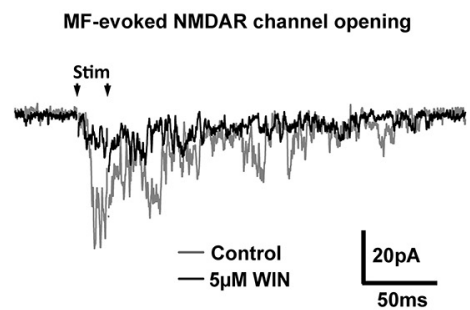

G

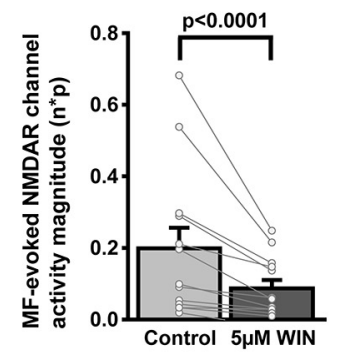

$\mathbf{H}$

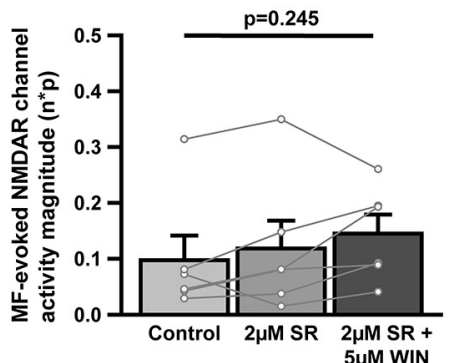

Figure 7. Activation of CB1Rs suppresses MF afferent-evoked stimulation of single NMDA receptor channels in nonsynaptically connected mGCs. $\boldsymbol{A}, \boldsymbol{B}$, Circuit diagram $(\boldsymbol{A})$ and DIC image ( $\boldsymbol{B})$ showing whole-cell patch recording of mGCs and location of stimulating electrode at glutamatergic MF afferents. C, Representative traces showing spontaneous and MF-evoked (Stim) single NMDAR channel openings and block by NMDAR antagonist AP5 $(50 \mu \mathrm{m})$, in voltage-clamped $\left(V_{\mathrm{h}}=-60 \mathrm{mV}\right) \mathrm{mGCs}$. D. Representative Gaussian fit of single-channel activity in an mGC. $\boldsymbol{E}$, Quantification of MF-evoked NMDAR channel activity $\left(n^{*} p\right)$ under control conditions and in the presence of AP5, showing that AP5 blocks MF-evoked NMDAR channel activity (paired Student's $t$ test, $t_{(3)}=4.6, p=0.02, n=4[4$ rats]). $\boldsymbol{F}$ - $\boldsymbol{H}$, Representative traces $(\boldsymbol{F})$ and quantification $(\boldsymbol{G}, \boldsymbol{H})$ of MF-evoked (Stim) NMDAR channel openings in $\mathrm{mGC}$, showing that WIN $(5 \mu \mathrm{m})$ significantly reduces NMDAR response magnitude under control conditions (G; Wilcoxon Signed Rank test, $z=-3.2, p<0.0001, n=13$ [5 rats]), but not in the presence of SR (H; $2 \mu \mathrm{m}$; repeated-measures ANOVA, $F_{(2)}=1.6, p=0.245, n=6[4$ rats $\left.]\right)$.

by nonsynaptically generated ambient extracellular glutamate (Rossi and Slater, 1993), given that exogenous/experimental modulation of mGC NMDAR activity levels controls migration rate, it is often suggested/assumed that afferent activity would modulate ambient glutamate concentrations, presumably to synchronize mGC migration rate with upstream afferent synaptic activity. However, to our knowledge, this has never been directly tested. We found that electrical stimulation of MF afferents (via a 
glass electrode placed in the white matter track; mean stimulus $=30-500 \mu \mathrm{A}$; Fig. $7 A, B)$ caused a transient but significant increase in mGC single NMDAR channel activity (\% increase = $1 \times 10^{9}$, paired Student's $t$ test, $t_{(3)}=-4.6, p=0.02$; Fig. $7 C$ ), that was blocked by AP5 (50 $\mu$; Fig. $7 E)$. Bath application of WIN (5 $\mu \mathrm{M})$ significantly reduced the magnitude of the MF-evoked increase in NMDAR channel activity (\% reduction $=57.7 \pm 9.3$; Fig. $7 F, G)$. Suppression of MF-evoked mGC NMDAR channel activity by WIN was blocked by the CB1R antagonist, SR141716A (SR; $2 \mu \mathrm{M}$; Fig. $7 H$ ), confirming its mediation by CB1Rs. Thus, the mGC NMDAR channel activity that drives their migration rate is stimulated by MF afferent activity, both of which are reduced by activation of CB1Rs on MF terminals.

Collectively, our data suggest that CB1Rs transiently expressed on MF afferent terminals enable coordinated modulation of afferent activity with trans-network developmental signaling, including MF excitation of GCs, and downstream GC excitation of developing PCs and mGCs. Given the importance of these developmental processes for proper cerebellar development and function, we next wanted to determine how the CB1R system is activated physiologically, and how such activation manifests (Fig. 8). As a starting point for such assessment, we implemented a common membrane depolarization protocol that has been shown to recruit endocannabinoid generation and consequent suppression of synaptic transmission known as depolarizationinduced suppression of inhibition/excitation (Yoshida et al., 2002; Diana and Marty, 2004). More specifically, at many synapses throughout the brain, including the adult cerebellum, depolarization of postsynaptic neurons raises intracellular $\left[\mathrm{Ca}^{2+}\right]$, and the resultant stimulation of postsynaptic endocannabinoid production, and diffusion to presynaptic terminal CB1Rs results in a transient suppression of vesicular release, and thus synaptic current magnitude (Yoshida et al., 2002). To determine whether a similar process occurs at MF to GC synapses in the developing cerebellum, we designed a protocol in which MF afferents were stimulated at $1 \mathrm{~Hz}$; and after recording synaptic responses to 60 such stimuli, stimulation was paused for $10 \mathrm{~s}$, and the postsynaptic GC was depolarized to $0 \mathrm{mV}$ for $100 \mathrm{~ms}, 10$ times (with $900 \mathrm{~ms}$ intervals between depolarizations; Fig. 8A). After the depolarization protocol, MF afferent stimulation at $1 \mathrm{~Hz}$ was reinitiated (for 60 stimulations), then paused for $10 \mathrm{~s}$ without depolarization, whereupon the sequence was repeated continuously under control conditions and then on bath application of the CB1R antagonist, SR $(2 \mu \mathrm{m}$; Fig. $8 A-C)$. The relatively high $\mathrm{MF}$ afferent stimulation frequency $(1 \mathrm{~Hz})$ was used because we needed better temporal resolution given high trial-to-trial variability in synaptic amplitude (Fig. 8B). In contrast to what has been reported previously in various adult brain regions, the depolarization protocol did not result in transient suppression of eEPSC amplitude (Fig. 8B,C). Despite the lack of depolarizationinduced suppression of excitation, we nonetheless applied SR to determine whether CB1Rs played any role in the observed temporal dynamics of synaptic response amplitude at these developing GC synapses (Fig. 8B-E). Although application of SR did not significantly affect temporal dynamics within a block of trials (Fig. 8C), it did lead to a significant, progressive, and sustained enhancement of eEPSC amplitude across all phases of our stimulation protocol (Fig. $8 D, E$ ). Such potentiation did not occur when the stimulation protocol was administered for the same duration without applying SR (Fig. $8 F, G$ ), confirming that the potentiation is specifically triggered by our stimulation protocol, but is normally suppressed, by concomitant CB1R activation. Thus, in developing neonatal cerebellar GCs, when CB1Rs are transiently expressed on afferent MF terminals, a typical depolarization-induced suppression of excitation protocol does activate the endogenous endocannabinoid system, but the outcome is atypical, resulting in an apparent suppression of progressive, sustained potentiation of synaptic transmission, which is revealed on blockade of CB1Rs.

\section{Discussion}

Our data provide previously unknown insight into five major aspects of cerebellar development: (1) there is a transient period in the newborn rodent, during the cerebellar "growth spurt," when functional CB1Rs are expressed on MF afferent terminals and on inhibitory interneuron cross-connected synapses, but not on the excitatory or inhibitory afferents to PCs that dominate in the adult; (2) despite the premature state of the cerebellar cortical network, adequate synaptic network connectivity exists, such that MF afferent excitatory activity is faithfully transmitted to downstream, cortical output PCs; (3) such MF-driven downstream synaptic activity releases enough glutamate to activate the NMDARs on synaptically unconnected, mGCs; (4) both MFdriven $\mathrm{PC}$ and $\mathrm{mGC}$ excitation is suppressed by activation of MF CB1Rs; and (5) endogenous activation of MF CB1Rs during GC excitation prevents progressive sustained synaptic potentiation.

To ensure that the lack of action of WIN on PC synapses in newborn cerebellum is indeed a developmentally transient phenomenon, we replicated past studies showing that WIN does effectively suppress the magnitude of parallel fiber to PC synaptic transmission in adult cerebellum (Fig. 1B) (Takahashi and Linden, 2000). The developmentally transient lack of functional CB1Rs on parallel fibers extends to synapses onto ML stellate and basket cells, which like PCs have functional CB1Rs in adult (Beierlein and Regehr, 2006) but not newborn cerebellum (Fig. $4 I, J)$. Since we are not aware of any previous studies of WIN at MF to GC synapses in the adult, we also tested WIN effects at this synapse in adult, and found no significant effects (Fig. $5 H$ ), confirming its developmentally transient nature. Although more temporally detailed developmental studies will be required to determine exactly when the respective functional expression sites arrive and disappear, based on our studies and past literature, we can conclude that exclusion/lack of functional CB1Rs on glutamatergic inputs to PCs, stellate cells, and basket cells, as well as GABAergic inputs to PCs (Figs. 3, 4), occurs during a short developmental window from birth to about PND 10-15 in rodents (Takahashi and Linden, 2000; Yoshida et al., 2002; Beierlein and Regehr, 2006). Based on anatomic comparisons, this rodent time frame is roughly equivalent to the third trimester of gestation through $\sim 8$ months after birth in humans (Zecevic and Rakic, 1976; Abraham et al., 2001). Conversely, although less temporally detailed, from data reported here, the transient expression of functional CB1Rs on MF terminals is gone by PND 35 in rodents (i.e., early adolescence) (Fig. $5 \mathrm{H}$ ).

The implications of our study for the role of the endocannabinoid system in cerebellar development and modulation by exogenous cannabinoids pertain to numerous components, including the following: (1) all phases of GC development, (2) PC dendritic growth and synaptic pruning, and (3) stellate/basket cell maturation.

First, it is known that glutamatergic synaptic excitation of postmigratory GCs regulates survival/apoptosis, dendrite formation/pruning, and expression of critical GC-specific gene products crucial for normal GC synaptic signaling. In particular, developmental activation of GC NMDARs results in GC 


\section{A Stimulation Protocol}

\section{Repeat 9 times (add SR, $2 \mu \mathrm{M}$ between block 4\&5)}

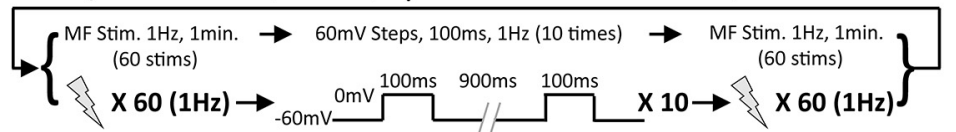

B
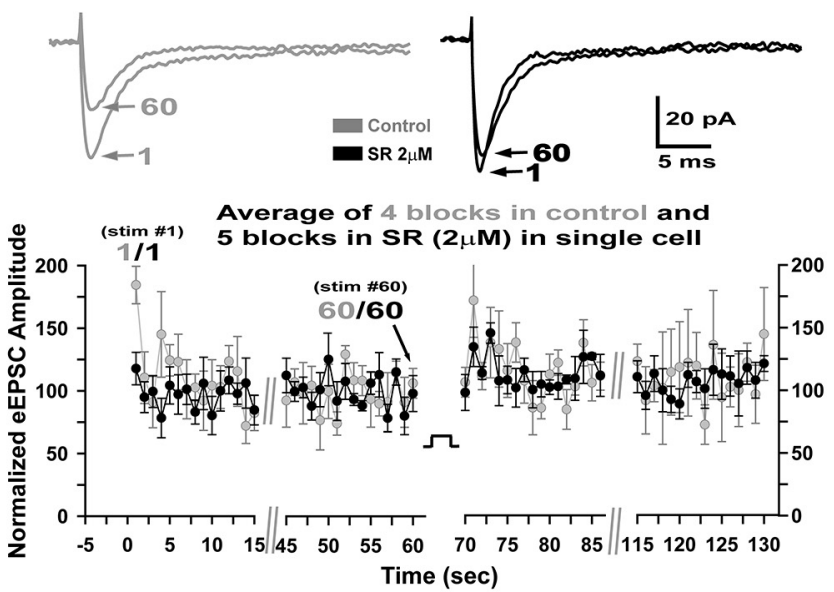

c

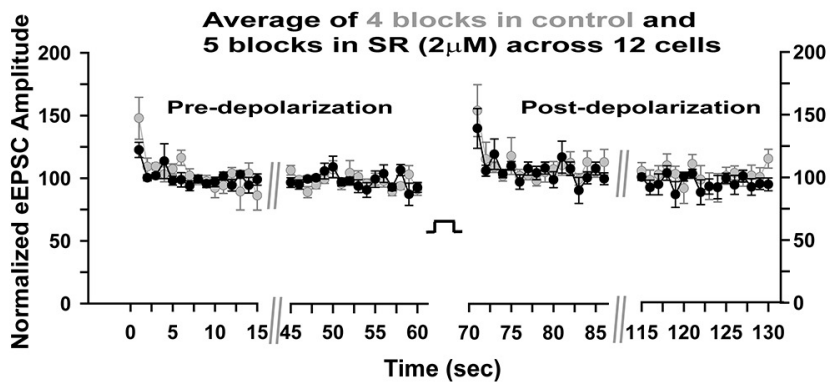

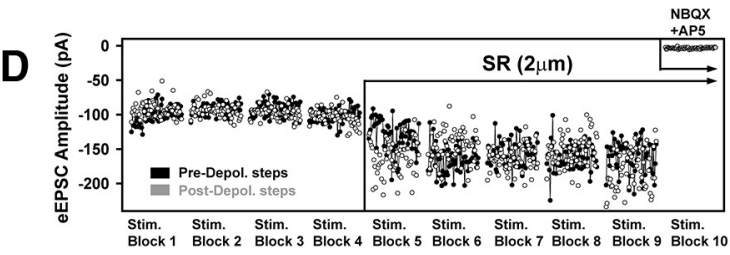

E

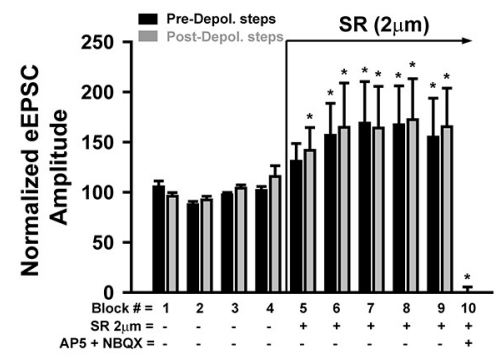

$F$

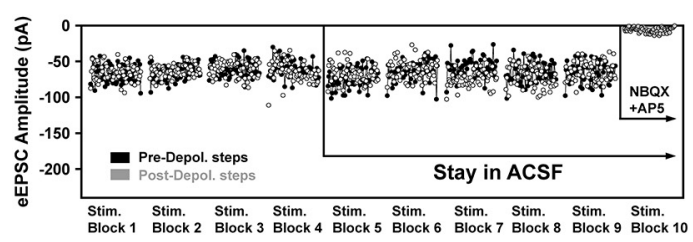

G

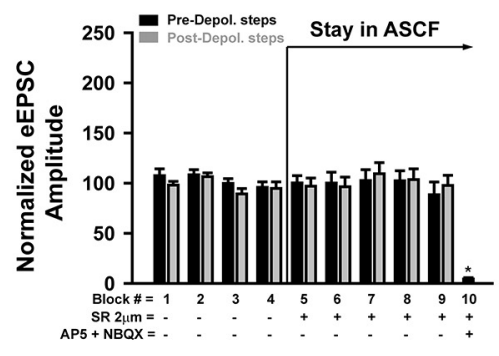

Figure 8. CB1R activation during synaptic trains and depolarization prevents progressive sustained potentiation. $A$, Graphical depiction of the synaptic stimulation and depolarization protocol designed to elicit endocannabinoid signaling. MF afferents were stimulated at $1 \mathrm{~Hz}$ for $60 \mathrm{~s}$ ( 60 total stimuli), followed by a depolarization protocol (to $0 \mathrm{mV}$ for $100 \mathrm{~ms}, 10$ times with $900 \mathrm{~ms}$ intervals) without synaptic stimulation (total 10 depolarization steps over $10 \mathrm{~s}$ ). Subsequently, MF afferent stimulation was renewed for another 60 stimuli, followed by a pause in stimulation without depolarization steps (for $10 \mathrm{~s}$ ). This protocol was repeated 10 times while varying pharmacological conditions. $\boldsymbol{B}$, Top, Raw data traces (top) showing the EPSC evoked by the first and 60th stimulation (average over 4 trials for the predepolarization stimulations under control conditions (left gray traces) and after application of SR ( $2 \mu \mathrm{m}$; right black traces). B, Bottom, Plot for a single cell (from which raw data traces were shown above) of mean eEPSC amplitude across 4 blocks of protocol under control conditions (gray dots) and 5 blocks of protocol in SR (black dots). C, Plot of mean eEPSC amplitude across cells $(n=12$ cells from 5 rats, PND 3-6) showing overall pattern of synaptic transmission during $1 \mathrm{~Hz}$ stimulation, and lack of effect of SR ( $2 \times 2$ repeated-measures ANOVA, condition $\times$ trial number interaction, $F_{(59,1298)}=1.01, p=0.45$; condition main effect, $F_{(1,22)}=0.46, p=0.51$; trial number main effect, $F_{(2.99,65.72)}=2.09$, $p=0.11) . \boldsymbol{D}, \boldsymbol{E}$, Plots of eEPSC amplitude across repetitions of the stimulation and depolarization protocol for representative cells $(\boldsymbol{D}, \boldsymbol{F})$ and means across cells $(\boldsymbol{E}, \boldsymbol{G})$ under control conditions throughout $\left(\boldsymbol{F}, \boldsymbol{G} ; n=8\right.$ cells from 5 rats, PND 3-5) or on transition to SR $\left(\boldsymbol{D}, \boldsymbol{E} ; n=12\right.$ cells from 5 rats PND 3-6). ${ }^{*} p<0.01$, pairwise comparisons for each block of trials in WIN, relative to the average of all trial blocks under control conditions (one-way repeated-measures ANOVA, $F_{(11,131)}=14.53, p<0.0001$, and follow-up Holm-Sidak, pairwise analysis indicated that all means for block 6-9, and the postdepolarization branch of block 5 were significantly different from the mean of the control blocks. Sequential $t$ scores and $p$ values are as follows: $t_{(9)}=2.4$, $p=0.009 ; t_{(9)}=3.7, p=0.003 ; t_{(9)}=4.2, p=0.003 ; t_{(9)}=4.2, p=0.001 ; t_{(9)}=4.6, p=0.002 ; t_{(9)}=4.4, p=0.003 ; t_{(9)}=4.3, p=0.003 ; t_{(9)}=4.2, p=0.003 ; t_{(9)}=4.2, p=0.002 ;$ and $t_{(9)}$ $=4.4, p<0.0001$ for blockers, NBQX + AP5. When SR was not applied $(G)$, there was no significant effect of time (one-way repeated-measures ANOVA, $\left.F_{(10,84)}=1.10, p=0.37\right)$, but the trial in the presence of blockers (NBQX + AP5) was significantly reduced compared with the preceding block $\left(t_{(5)}=8.84, p<0.0001\right)$. Each block of $1 \mathrm{~Hz}$ stimulation from before $(\boldsymbol{D}, \boldsymbol{F}$, black dots; $\boldsymbol{E}, \boldsymbol{G}$, bars) and after the depolarization protocol $(\boldsymbol{D}, \boldsymbol{F}$, gray dots; $\boldsymbol{E}, \boldsymbol{G}$, bars) is overlaid on one another for each block. There is a $10 \mathrm{~s}$ gap between repetitions of each block. $\boldsymbol{D}-\boldsymbol{G}, \mathbf{T}, \mathrm{The}$ last block of protocol execution (block 10) was conducted in glutamate receptor antagonists, NBQX and AP5 (25 and $50 \mu \mathrm{m}$, respectively), which, in all cases eliminated synaptic responses, confirming that all eEPSCs were mediated by glutamate receptors.

production of BDNF, which in turn promotes GC survival (Hirai and Launey, 2000). Similarly, release of WNT-7a from developing GCs, possibly dependent on $\mathrm{Ca}^{2+}$ influx through activated NMDARs (Wayman et al., 2006), promotes the growth and synaptic maturation of both GC dendrites and incoming MFs (Ahmad-Annuar et al., 2006). In this regard, it appears that endogenous activation of MF CB1Rs during GC excitation retards a form of progressive synaptic potentiation that would occur in their absence (Fig. $8 D-G$ ), suggesting a key role in regulating synaptic maturation. Moreover, like most neurons, GCs exhibit developmental shifts in their expression of molecular subtypes of transmitter-gated ion channels (Nakanishi and Okazawa, 2006; Okazawa et al., 2009; Ding et al., 2013), including GC-specific subtypes of $\mathrm{GABA}_{\mathrm{A}} \mathrm{R}$ subunits (e.g., $\alpha 6$ ) that are critical for GC function in the adult (Hamann et al., 2002). Importantly, such transitions are regulated by GC excitation. Thus, activation of MF CB1Rs, which suppresses glutamatergic synaptic excitation of postmigratory GCs by incoming MFs (Figs. 5, 8), is likely to disrupt normal afferent-driven GC survival, synaptogenesis, and differentiation into an appropriate adult molecular phenotype. 
Second, previous work has shown that GC activity drives PC dendritic growth (Hirai and Launey, 2000) and is necessary for appropriate pruning of supernumerary climbing fiber inputs to PCs (Hashimoto et al., 2009). Our data demonstrate that CB1R suppression of MF to GC synaptic transmission also dampens MF-evoked downstream polysynaptic excitation of PCs (Fig. 6). Thus, despite the lack of functional CB1Rs on parallel fiber to PC synapses (Fig. 3), activation of MF-GC CB1Rs will likely alter afferent-driven polysynaptic excitation of PCs, which could therefore also hamper appropriate PC dendritic growth and synaptic maturation. Furthermore, our data demonstrate that afferent MF excitation of postmigratory GCs increases the ambient concentration of glutamate in the developing ML enough to substantially increase the nonsynaptic NMDAR channel activity in mGCs that is known to drive their migration rate (Fig. 7) (Komuro and Rakic, 1993). Thus, CB1R-induced suppression of MF afferent to GC transmission is also likely to reduce mGC migration rate.

Finally, although less well studied, analysis of mutant lines and $\mathrm{x}$-ray-irradiated rodents that result in GC loss suggest that normal stellate and basket cell dendritic growth is dependent on innervation from GCs, either directly or via GC driven crosstalk among interneurons (Rakic and Sidman, 1973; Altman, 1976). Thus, it is reasonable to consider that CB1R suppression of MF to GC excitation (Fig. 5) and/or interneuron crosstalk (Fig. 4I,J) will adversely affect ML interneuronal dendritic development. Future research will be required to determine which of all these likely impacts occurs, to what degree they occur under various conditions, and how they relate to the long-lasting effect of perinatal exposure to cannabinoids on motor learning.

Collectively, our data demonstrate that numerous cerebellar cortical developmental signals are modified in a coordinated fashion by MF fiber afferent excitation of GCs, and that activation of CB1Rs on MF terminals consequently dampens all of the respective coordinated signal cascades. While we have used a saturating concentration of the CB1R agonist to study such processes (WIN, $5 \mu \mathrm{M}$ ), and maximal CB1R activation is not likely common during recreational use of cannabis, our data nonetheless suggest that cannabinoid use by women during the third trimester of pregnancy and first year of breastfeeding have great potential for disrupting cerebellar development in the exposed offspring. Such exposure may account for established impacts of marijuana use by pregnant women on motor skills in their offspring (Astley and Little, 1990; Willford et al., 2010; Campolongo et al., 2011), but may also either directly induce nonmotor, cognitive symptoms that are known to occur in cerebellar-associated neurodevelopmental cognitive disorders (attention deficit and hyperactivity disorder, schizophrenia, autism spectrum disorder, and predilection to drug/alcohol abuse) (Kaplan et al., 2013; Stoodley, 2016; Hariri, 2019), or synergize with respective genetic risks for such conditions. Alternatively, our increasing understanding of the genetics that drive such developmental disorders may reveal potential developmental therapeutic applications of CB1R activation during the cerebellar growth spurt.

\section{References}

Abraham H, Tornoczky T, Kosztolanyi G, Seress L (2001) Cell formation in the cortical layers of the developing human cerebellum. Int J Dev Neurosci 19:53-62.

Ahmad-Annuar A, Ciani L, Simeonidis I, Herreros J, Fredj NB, Rosso SB, Hall A, Brickley S, Salinas PC (2006) Signaling across the synapse: a role for Wnt and Dishevelled in presynaptic assembly and neurotransmitter release. J Cell Biol 174:127-139.
Altman J (1976) Experimental reorganization of the cerebellar cortex: VII. Effects of late $\mathrm{x}$-irradiation schedules that interfere with cell acquisition after stellate cells are formed. J Comp Neurol 165:65-75.

Astley SJ, Little RE (1990) Maternal marijuana use during lactation and infant development at one year. Neurotoxicol Teratol 12:161-168.

Beierlein M, Regehr WG (2006) Local interneurons regulate synaptic strength by retrograde release of endocannabinoids. J Neurosci 26:99359943.

Bodranghien F, Bastian A, Casali C, Hallett M, Louis ED, Manto M, Marien P, Nowak DA, Schmahmann JD, Serrao M, Steiner KM, Strupp M, Tilikete C, Timmann D, van Dun K (2016) Consensus paper: revisiting the symptoms and signs of cerebellar syndrome. Cerebellum 15:369-391.

Brown SP, Brenowitz SD, Regehr WG (2003) Brief presynaptic bursts evoke synapse-specific retrograde inhibition mediated by endogenous cannabinoids. Nat Neurosci 6:1048-1057.

Campolongo P, Trezza V, Ratano P, Palmery M, Cuomo V (2011) Developmental consequences of perinatal cannabis exposure: behavioral and neuroendocrine effects in adult rodents. Psychopharmacology (Berl) 214:5-15.

DeSanty KP, Dar MS (2001) Cannabinoid-induced motor incoordination through the cerebellar $\mathrm{CB}(1)$ receptor in mice. Pharmacol Biochem Behav 69:251-259.

Dhar M, Hantman AW, Nishiyama H (2018) Developmental pattern and structural factors of dendritic survival in cerebellar granule cells in vivo. Sci Rep 8:17561.

Diana MA, Marty A (2004) Endocannabinoid-mediated short-term synaptic plasticity: depolarization-induced suppression of inhibition (DSI) and depolarization-induced suppression of excitation (DSE). Br J Pharmacol 142:9-19.

Diana MA, Levenes C, Mackie K, Marty A (2002) Short-term retrograde inhibition of GABAergic synaptic currents in rat Purkinje cells is mediated by endogenous cannabinoids. J Neurosci 22:200-208.

Díaz-Alonso J, Aguado T, Wu CS, Palazuelos J, Hofmann C, Garcez P, Guillemot F, Lu HC, Lutz B, Guzmán M, Galve-Roperh I (2012a) The CB (1) cannabinoid receptor drives corticospinal motor neuron differentiation through the Ctip2/Satb2 transcriptional regulation axis. J Neurosci 32:16651-16665.

Díaz-Alonso J, Guzmán M, Galve-Roperh I (2012b) Endocannabinoids via $\mathrm{CB}(1)$ receptors act as neurogenic niche cues during cortical development. Philos Trans R Soc Lond B Biol Sci 367:3229-3241.

Ding B, Wang W, Selvakumar T, Xi HS, Zhu H, Chow CW, Horton JD, Gronostajski RM, Kilpatrick DL (2013) Temporal regulation of nuclear factor one occupancy by calcineurin/NFAT governs a voltage-sensitive developmental switch in late maturing neurons. J Neurosci 33:28602872.

Duff G, Argaw A, Cecyre B, Cherif H, Tea N, Zabouri N, Casanova C, Ptito M, Bouchard JF (2013) Cannabinoid receptor CB2 modulates axon guidance. PLoS One 8:e70849.

Ebralidze AK, Rossi DJ, Tonegawa S, Slater NT (1996) Modification of NMDA receptor channels and synaptic transmission by targeted disruption of the NR2C gene. J Neurosci 16:5014-5025.

Feller MB (1999) Spontaneous correlated activity in developing neural circuits. Neuron 22:653-656.

Galas L, Benard M, Lebon A, Komuro Y, Schapman D, Vaudry H, Vaudry D, Komuro H (2017) Postnatal migration of cerebellar interneurons. Brain Sci 7:62.

Hamann M, Rossi DJ, Attwell D (2002) Tonic and spillover inhibition of granule cells control information flow through cerebellar cortex. Neuron 33:625-633.

Hariri AR (2019) The emerging importance of the cerebellum in broad risk for psychopathology. Neuron 102:17-20.

Hashimoto K, Kano M (2013) Synapse elimination in the developing cerebellum. Cell Mol Life Sci 70:4667-4680.

Hashimoto K, Yoshida T, Sakimura K, Mishina M, Watanabe M, Kano M (2009) Influence of parallel fiber-Purkinje cell synapse formation on postnatal development of climbing fiber-Purkinje cell synapses in the cerebellum. Neuroscience 162:601-611.

Herkenham M, Lynn AB, Johnson MR, Melvin LS, de Costa BR, Rice KC (1991) Characterization and localization of cannabinoid receptors in rat brain: a quantitative in vitro autoradiographic study. J Neurosci 11:563583. 
Higuera-Matas A, Ucha M, Ambrosio E (2015) Long-term consequences of perinatal and adolescent cannabinoid exposure on neural and psychological processes. Neurosci Biobehav Rev 55:119-146.

Hirai H, Launey T (2000) The regulatory connection between the activity of granule cell NMDA receptors and dendritic differentiation of cerebellar Purkinje cells. J Neurosci 20:5217-5224.

Kano M, Watanabe T, Uesaka N, Watanabe M (2018) Multiple phases of climbing fiber synapse elimination in the developing cerebellum. Cerebellum 17:722-734.

Kaplan JS, Mohr C, Rossi DJ (2013) Opposite actions of alcohol on tonic GABA(A) receptor currents mediated by nNOS and PKC activity. Nat Neurosci 16:1783-1793.

Kirkby LA, Sack GS, Firl A, Feller MB (2013) A role for correlated spontaneous activity in the assembly of neural circuits. Neuron 80:1129-1144.

Kishimoto Y, Kano M (2006) Endogenous cannabinoid signaling through the $\mathrm{CB} 1$ receptor is essential for cerebellum-dependent discrete motor learning. J Neurosci 26:8829-8837.

Komuro H, Rakic P (1993) Modulation of neuronal migration by NMDA receptors. Science 260:95-97.

Leto K, Arancillo M, Becker EB, Buffo A, Chiang C, Ding B, Dobyns WB, Dusart I, Haldipur P, Hatten ME, Hoshino M, Joyner AL, Kano M, Kilpatrick DL, Koibuchi N, Marino S, Martinez S, Millen KJ, Millner TO, Miyata T, et al. (2016) Consensus paper: cerebellar development. Cerebellum 15:789-828.

Maccarrone M, Guzman M, Mackie K, Doherty P, Harkany T (2014) Programming of neural cells by (endo)cannabinoids: from physiological rules to emerging therapies. Nat Rev Neurosci 15:786-801.

Matsuda LA, Bonner TI, Lolait SJ (1993) Localization of cannabinoid receptor mRNA in rat brain. J Comp Neurol 327:535-550.

Mayer ML, Westbrook GL, Guthrie PB (1984) Voltage-dependent block by $\mathrm{Mg}^{2+}$ of NMDA responses in spinal cord neurones. Nature 309:261-263.

Mugnaini E (1970) The relation between cytogenesis and the formation of different types of synaptic contact. Brain Res 17:169-179.

Nakanishi S, Okazawa M (2006) Membrane potential-regulated $\mathrm{Ca}^{2+}$ signalling in development and maturation of mammalian cerebellar granule cells. J Physiol (Lond) 575:389-395.

Okazawa M, Abe H, Katsukawa M, Iijima K, Kiwada T, Nakanishi S (2009) Role of calcineurin signaling in membrane potential-regulated maturation of cerebellar granule cells. J Neurosci 29:2938-2947.

Rakic P, Sidman RL (1973) Organization of cerebellar cortex secondary to deficit of granule cells in weaver mutant mice. J Comp Neurol 152:133161.

Ramaekers JG, Kauert G, van Ruitenbeek P, Theunissen EL, Schneider E, Moeller MR (2006) High-potency marijuana impairs executive function and inhibitory motor control. Neuropsychopharmacology 31:2296-2303.

Rossi DJ, Slater NT (1993) The developmental onset of NMDA receptorchannel activity during neuronal migration. Neuropharmacology 32:1239-1248.
Saez TM, Aronne MP, Caltana L, Brusco AH (2014) Prenatal exposure to the CB1 and CB2 cannabinoid receptor agonist WIN 55,212-2 alters migration of early-born glutamatergic neurons and GABAergic interneurons in the rat cerebral cortex. J Neurochem 129:637-648.

Safo PK, Cravatt BF, Regehr WG (2006) Retrograde endocannabinoid signaling in the cerebellar cortex. Cerebellum 5:134-145.

Schilling K (2018) Moving into shape: cell migration during the development and histogenesis of the cerebellum. Histochem Cell Biol 150:13-36.

Sonego M, Gajendra S, Parsons M, Ma Y, Hobbs C, Zentar MP, Williams G, Machesky LM, Doherty P, Lalli G (2013) Fascin regulates the migration of subventricular zone-derived neuroblasts in the postnatal brain. J Neurosci 33:12171-12185.

Stoodley CJ (2016) The cerebellum and neurodevelopmental disorders. Cerebellum 15:34-37.

Suarez J, Bermudez-Silva FJ, Mackie K, Ledent C, Zimmer A, Cravatt BF, de Fonseca FR (2008) Immunohistochemical description of the endogenous cannabinoid system in the rat cerebellum and functionally related nuclei. J Comp Neurol 509:400-421.

Takahashi KA, Linden DJ (2000) Cannabinoid receptor modulation of synapses received by cerebellar Purkinje cells. J Neurophysiol 83:1167-1180.

Trazzi S, Steger M, Mitrugno VM, Bartesaghi R, Ciani E (2010) CB1 cannabinoid receptors increase neuronal precursor proliferation through AKT/ glycogen synthase kinase-3beta/beta-catenin signaling. J Biol Chem 285:10098-10109.

Tsou K, Brown S, Sanudo-Pena MC, Mackie K, Walker JM (1998) Immunohistochemical distribution of cannabinoid CB1 receptors in the rat central nervous system. Neuroscience 83:393-411.

Wayman GA, Impey S, Marks D, Saneyoshi T, Grant WF, Derkach V, Soderling TR (2006) Activity-dependent dendritic arborization mediated by CaM-kinase I activation and enhanced CREB-dependent transcription of Wnt-2. Neuron 50:897-909.

Willford JA, Chandler LS, Goldschmidt L, Day NL (2010) Effects of prenatal tobacco, alcohol and marijuana exposure on processing speed, visualmotor coordination, and interhemispheric transfer. Neurotoxicol Teratol 32:580-588.

Yamasaki M, Hashimoto K, Kano M (2006) Miniature synaptic events elicited by presynaptic $\mathrm{Ca}^{2+}$ rise are selectively suppressed by cannabinoid receptor activation in cerebellar Purkinje cells. J Neurosci 26:86-95.

Yoshida T, Hashimoto K, Zimmer A, Maejima T, Araishi K, Kano M (2002) The cannabinoid CB1 receptor mediates retrograde signals for depolarization-induced suppression of inhibition in cerebellar Purkinje cells. J Neurosci 22:1690-1697.

Zecevic N, Rakic P (1976) Differentiation of Purkinje cells and their relationship to other components of developing cerebellar cortex in man. J Comp Neurol 167:27-47.

Zimmer A, Zimmer AM, Hohmann AG, Herkenham M, Bonner TI (1999) Increased mortality, hypoactivity, and hypoalgesia in cannabinoid CB1 receptor knockout mice. Proc Natl Acad Sci USA 96:5780-5785. 